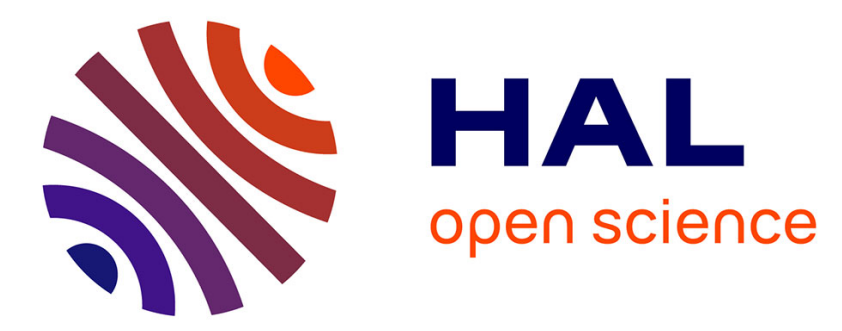

\title{
A one-dimensional full-range two-phase model to efficiently compute bifurcation diagrams in sub-cooled boiling flows in vertical heated tube
}

\author{
Marc Medale, Bruno Cochelin, Edouard Bissen, Nicolas Alpy
}

\section{To cite this version:}

Marc Medale, Bruno Cochelin, Edouard Bissen, Nicolas Alpy. A one-dimensional full-range two-phase model to efficiently compute bifurcation diagrams in sub-cooled boiling flows in vertical heated tube. Journal of Computational Physics, 2020, 404, 10.1016/j.jcp.2019.109131 hal-02436969

\section{HAL Id: hal-02436969 \\ https://hal-amu.archives-ouvertes.fr/hal-02436969}

Submitted on 13 Jan 2020

HAL is a multi-disciplinary open access archive for the deposit and dissemination of scientific research documents, whether they are published or not. The documents may come from teaching and research institutions in France or abroad, or from public or private research centers.
L'archive ouverte pluridisciplinaire HAL, est destinée au dépôt et à la diffusion de documents scientifiques de niveau recherche, publiés ou non, émanant des établissements d'enseignement et de recherche français ou étrangers, des laboratoires publics ou privés. 


\title{
A one-dimensional full-range two-phase model to efficiently compute bifurcation diagrams in sub-cooled boiling flows in vertical heated tube
}

\author{
Marc Medale $^{\mathrm{a}, *}$, Bruno Cochelin ${ }^{\mathrm{b}}$, Edouard Bissen ${ }^{\mathrm{a}, \mathrm{c}}$, Nicolas Alpy ${ }^{\mathrm{c}}$ \\ a Aix-Marseille Université and IUSTI, UMR 7343 CNRS, 5 rue Enrico Fermi, Technopole de Chateau-Gombert, 13453 Marseille Cedex 13, France \\ b Aix Marseille univ, CNRS, Centrale Marseille, LMA UMR CNRS 7051, France \\ c CEA, DEN, DER, SESI, LEMS, 13108 Saint Paul Lez Durance, France
}

Keywords:

Liquid-vapor two-phase fluid flows

1D Drift flux model

Asymptotic Numerical Method

Bifurcation diagrams

Hopf bifurcation

Density wave instabilities

\begin{abstract}
A B S T R A C T
This paper presents a powerful numerical model to compute bifurcation diagrams in liquidvapor two-phase fluid flows in vertical heated tube. This full range two-phase model is designed to deal with both single phase (purely liquid or purely vapor) and mixed liquid-vapor configurations that span all flow regimes (laminar and turbulent) in forced, mixed and natural convections. The originality of the proposed methodology is to faithfully integrate the implicit highly nonlinear system of governing equations along branches of steady-state solutions. This is performed by means of a continuation algorithm based on the Asymptotic Numerical Method supplemented with Automatic Differentiation. Then, linear stability analyses are performed at various points of interest, enabling to figure out stability limits within the parameter space in natural circulation configurations. Markedly, Hopf bifurcations that indicate limit-cycle occurrences are identified at low and medium void fractions, respectively, showing the added-value of the approach to track density-wave mechanisms and potential failure of standard application of Ledinegg stability criteria on such cases.
\end{abstract}

\section{Introduction}

In-depth understanding of two-phase natural circulation systems is crucial to design reliable industrial components likely to be used as passive safety systems in various environments such as the next generation of nuclear reactor power plants [26,40]. Indeed, natural circulation or convection fluid flow sets in a gravity field provided density variations occur over any equal elevation plane. In most cases, density variations result from temperature, concentration or pressure differences, so the central feature of such intrinsic behavior is that fluid flows without any actuator or pump. This is the main reason for which natural circulation loops could be good candidates for passive safety systems. However, despite their apparent simplistic design, two-phase natural circulation loops could be very difficult to optimally design over a wide range of operating conditions owing to the many instabilities that may develop.

As a matter of fact, boiling channels are notoriously prone to thermal-hydraulic instabilities $[4,46]$ and density-wave instabilities [21] are the most common type of instabilities that occurs even in the simplest configuration of a single vertical

\footnotetext{
* Corresponding author.

E-mail address: marc.medale@univ-amu.fr (M. Medale).
} 
tube uniformly heated along its wall $[1,28,30,55,59]$. However, to design reliable passive safety systems all instabilities should be either avoided or at least moderated to prevent boiling crisis or dry-out. Indeed, these two effects are unavoidably associated with reduced heat transfer between the cooling flow and heating surfaces possibly leading to wall temperature elevation likely to irreversibly damage the system. Specifically, when the passive system leads to a periodic flow pattern resulting from a density wave mechanism [3], stability of the limit cycle should be demonstrated, which first requires to identify the likely related Hopf bifurcation. However, despite several decades of intense research activity, experimental or numerical stability analyses of boiling fluid flows are still a very challenging research area. Undeniably, these instabilities results from complex interplay between various physical mechanisms acting at different space and time scales $[4,21,46]$.

To model such a wide range of instabilities from static to dynamic ones, one needs a robust and representative numerical model suitable to deal with subtle highly nonlinear phenomena at affordable computational costs. The governing equations are derived on the one hand from conservation equations (mass, momentum and energy) and on the other hand from constitutive relationships that provide closures leading to a solvable set of equations. Provided the multiplicity of existing models in the open scientific literature only few leading one-dimensional approaches for boiling channel flows are considered here. The three conservation equations can be derived for each phase (liquid and vapor), leading to six conservation equations. These can be combined to end up in three conservation equations for the two-phase mixture, depending on the ability to model interaction terms linking liquid and vapor phases across their interfaces and at solid walls [58]. These are the two main issues to overcome when dealing with one-dimensional boiling channel models. Homogeneous models [1,30, 55] assume no relative velocity between liquid and vapor phases. Therefore, they should be used only when the two-phase flows that contains bubbles or drops among the continuous phases can be assumed sufficiently smoothly varying. Otherwise, drift-flux models $[28,59]$ are to be preferred as they account for some averaged relative velocities between vapor and liquid phases. Countless variants using sophisticated tailored correlations have been introduced over four decades to account for particular situations such as pattern or regime transitions $[18,24,25,42,47,50]$. The second main issue is related to two-phase frictional pressure drop as it also remains a scientific question not entirely resolved yet. Numerous empirical correlations have been proposed to cover a broad range of configurations [51,57] and surprisingly the Muller-Steinhagen constitutive relationship [37] performs among the best, despite being one of the simplest. So, boiling channel flows involve many phenomenological closures or constitutive relationships that make up the set of governing equations. Unfortunately for real life industrial applications these governing equations translate into stiff partial differential equations (PDEs) requiring out off the shelves specialized numerical methods and models to compute fully nonlinear base-state solutions and perform their stability analyses.

The overall goal of stability analyses in the framework of nonlinear problems is to understand the complete behavior of a given system as a function of its parameters. Relevant questions are: How many steady-states are there? Are they stable or unstable? It is important to have the ability to compute unstable steady-states as well as stable ones, since solutions arising from bifurcations along unstable branches often interact with stable solutions producing otherwise inexplicable phenomena. Two main numerical approaches can be used to answer some of the above questions [14]. In a first approach the time-dependent PDEs are discretized in space and the resulting system of ordinary differential equations (ODEs) is evolved forward in time for various fixed values of the parameters. This approach is the most widespread approach to investigate the linear stability of boiling flows in heated channels. It originates from semi-analytical models in the 1960s in which the linearization of PDEs governing equations to transform them into ODEs was performed thanks to Laplace transforms [30, 55], lumped parameter methods [1,47] or reduced order models [31]. These semi-analytical approaches enabled to perform linear stability analyses that contributed to significant breakthroughs in understanding the numerous boiling channel instabilities. However, the main issue of these approaches is that the derivation of the various transfer function coefficients is cumbersome and can be done only under very restrictive conditions either on base-state solutions or equations for which the linearization is performed. The alternative approach is to discretize the steady problem to obtain a system of nonlinear equations and then use methods from nonlinear analyses $[27,29,32]$ to compute paths of steady solutions and provide stability assignment using numerical continuation methods and eigenvalue information [15-17,29,48,49]. This latter approach enables to overcome the issue of formal derivation of transfer function coefficients, but usually at much higher computational costs compared to previous semi-analytical models.

The present work aims at contributing to a better understanding of boiling channel instabilities by the means of a numerical model that takes full advantage of robust and efficient advanced semi-analytical methods based on the Asymptotic Numerical Method [12,13,34]. This method is particularly well-suited to highly nonlinear problems such as those encountered in boiling channels flows. This nevertheless requires the governing equations to be somehow recast in the framework of high-order model requirements. Indeed, continuity and derivability properties are necessary conditions to compute accurately and efficiently high-order Taylor series expansions involved in the Asymptotic Numerical Method. To this aim, we have derived a set of drift-flux based governing equations made up of three conservation equations for the two-phase mixture, supplemented with a specifically derived void fraction equation, along with general-purpose constitutive laws for the relative liquid-vapor velocity and two-phase pressure drop. The present approach follows the Dynamical System Theory in the way one first computes branches of fixed points, i.e., steady-state solutions of the governing equations, thanks to the high-order continuation algorithm and then use some of these fixed point solutions as base-state to perform linear stability analyses by solving generalized eigenvalue problems. The implementation is done in the framework of the Diamanlab software [5,6] and the outcome is the Baccarat model, which stands for Bifurcation Analysis in a vertiCal Channel by the Asymptotic numeRicAl meThod. Numerical assessments of the developed model are first presented. 
The paper is organized as follows. The governing equations for boiling flows in vertical heated tube are derived in Section 2. Then, in Section 3, the developed numerical model is presented along with numerical model assessments in Section 4. Finally, conclusions and future work directions are given in Section 5, followed by three appendices dedicated to specific parts of the developed model.

\section{Governing equations}

The one-dimensional model developed in this work is intended to account for two-phase flows (liquid-vapor phasechange) in a vertical tube (length $l$, constant cross-section of diameter $d$ ), heated at its wall with a prescribed heat flux $\left(q_{w}\right)$. The fluid flow can be driven by an external pressure head and $\backslash$ or buoyancy so that one can model various interesting configurations ranging from forced convection to natural circulation and from laminar to turbulent regimes.

The goals implying the way we have derived the governing equations are twofold: i) to achieve a full-range two-phase model that enables to deal with both single phase (purely liquid or purely vapor) and mixed liquid-vapor flows that span laminar and turbulent regimes in forced, mixed or natural circulation; ii) the resulting set of equations should satisfy continuity and derivability constraints required by the solution algorithm based on the Asymptotic Numerical Method, which internally computes, transforms and manages high-order Taylor series expansions.

\subsection{Conservation equations}

\subsubsection{Mass conservation equation}

Let us define $\rho_{m}$ and $G$ as cross-sectional averages of mixture density and mass flux (mass flow rate per area unit), respectively, according to the following relationships:

$$
\begin{aligned}
\rho_{m} & =(1-\alpha) \rho_{l}+\alpha \rho_{v} \\
G & =(1-\alpha) \rho_{l} u_{l}+\alpha \rho_{v} u_{v}
\end{aligned}
$$

where $\alpha$ is the cross-sectional average fraction of vapor phase (often referred to as void fraction), $\rho_{i}$ is $i$-fluid phase density ( $i=l, v$ liquid or vapor phase, respectively) and $u_{i}$ is its one-dimensional cross-sectional average velocity. Then, the one-dimensional mass conservation equation of the liquid-vapor mixture reads (cf. eq. (A.1)-(A.4) for derivation details):

$$
\frac{\partial \rho_{m}}{\partial t}+\frac{\partial G}{\partial z}=0
$$

where $t$ is time and $z$ the axial abscissa along the ascending vertical axis.

\subsubsection{Momentum conservation equation}

The one-dimensional momentum equation of the two-phase liquid-vapor mixture in projection along the ascending vertical direction reads as follows (cf. eq. (A.5)-(A.10) for derivation details):

$$
\frac{\partial G}{\partial t}+\frac{\partial}{\partial z}\left[\frac{G^{2}}{\rho_{m}}+\alpha(1-\alpha) \frac{\rho_{l} \rho_{v}}{\rho_{m}} U_{r_{v l}}^{2}\right]=-\frac{\partial p_{m}}{\partial z}-\left[(1-\beta) \tau_{l}+\beta \tau_{v}\right] \frac{4}{d}-\rho_{m} g
$$

where $U_{r_{v l}}=u_{v}-u_{l}$ is the relative liquid-vapor velocity, $p_{m}$ is the mixture pressure, $\beta$ is the perimeter fraction wetted by vapor phase, $\tau_{i}$ is the viscous stress of the $i$-fluid phase at tube wall and $g$ is gravity.

\subsubsection{Energy conservation equation}

Let us define $\rho_{m} h_{m}$ as cross-sectional average of mixture mass enthalpy, according to the following relationship:

$$
\rho_{m} h_{m}=(1-\alpha) \rho_{l} h_{l}+\alpha \rho_{v} h_{v}
$$

then, the one-dimensional enthalpy conservation equation of the liquid-vapor mixture reads (cf. eq. (A.12)-(A.20) for derivation details):

$$
\begin{aligned}
& \frac{\partial\left(\rho_{m} h_{m}-p_{m}\right)}{\partial t}+\frac{\partial}{\partial z}\left[G h_{m}+\alpha(1-\alpha) \frac{\rho_{l} \rho_{v}}{\rho_{m}} U_{r_{v l}} H_{r_{v l}}\right] \\
& -\left[\frac{G}{\rho_{m}}+\alpha(1-\alpha) \frac{\left(\rho_{l}-\rho_{v}\right)}{\rho_{m}} U_{r_{v l}}\right] \frac{\partial p_{m}}{\partial z}=q_{w} \frac{4}{d}-F_{i_{l v}} U_{r_{v l}}
\end{aligned}
$$

where $H_{r_{v l}}=h_{v}-h_{l}$ is the relative liquid-vapor enthalpy and $F_{i_{l v}}$ is the interaction force between phases acting at the liquid-vapor interface. 


\subsection{Closures for our full-range drift-flux model}

At this point, one has derived three conservation equations (mass eq. (3), momentum eq. (4) and enthalpy eq. (6)), but they involve twelve unknowns $\left(\rho_{l}, \rho_{v}, \alpha, G, p_{m}, h_{m}, U_{r_{v l}}, H_{r_{v l}}, \beta, \tau_{l}, \tau_{v}, F_{i_{l v}}\right)$. Therefore, nine extra equations (constitutive relationships, closures or assumptions) have to be supplemented to conservation equations to lead to a solvable set of equations.

\subsubsection{Negligible compressibility effects}

Let us first show that in the particular liquid-vapor two-phase flows we are interested in (steady-states and very lowdynamic subsonic flows, i.e., no water hammer), compressibility effects are negligible with respect to phase-change dilatation ones. Indeed, magnitude orders of the two related effects can be evaluated thanks to the total differential of mixture density as follows:

$$
d \rho_{m}=\frac{\partial \rho_{m}}{\partial p_{m}} \Delta p_{m}+\frac{\partial \rho_{m}}{\partial T} \Delta T
$$

where $T$ is temperature, the former term on the right hand side of eq. (7) represents compressibility effect meanwhile the latter represents the dilatation induced by internal energy variations (dominated by phase-change). In the particular cases we are interested in, the maximum vapor velocity does not overcome some fraction of the speed of sound in that fluid $\left(M a_{v}=u_{v} / c_{s v}<0.3, M a_{v}\right.$ being the Mach number in the vapor phase and $c_{s v} \approx 330 \mathrm{~ms}^{-1}$ is the speed of sound in the vapor at atmospheric pressure). So, one can estimate the compressibility contribution as follows: $\frac{\partial \rho_{m}}{\partial p_{m}}=\frac{\partial \rho_{l}}{\partial p_{m}}+\frac{\partial \rho_{v}}{\partial p_{m}} \approx \frac{1}{c_{s l}^{2}}+\frac{1}{c_{s v}^{2}} \approx$ $\frac{1}{c_{s v}^{2}}$, meanwhile the maximum pressure drop considered in the present work is $\Delta p=O\left(10^{5}\right) P a$. Thus, the compressibility contribution is $\frac{\partial \rho_{m}}{\partial p} \Delta p_{m}=O(1)$, whereas the dilatation one is $\frac{\partial \rho_{m}}{\partial T} \Delta T=O\left(\frac{\rho_{l}}{\rho_{v}}\right) \approx O\left(10^{3}\right)$. Therefore, $\frac{\partial \rho_{m}}{\partial p_{m}} \Delta p_{m}<<\frac{\partial \rho_{m}}{\partial T} \Delta T$, so that compressibility effects are legitimately negligible with respect to phase-change dilatation ones. So, in the following, one assumes liquid and vapor density to be user provided constants:

$$
\begin{gathered}
\rho_{l}=\rho_{l}\left(p_{\text {ref }}, T_{\text {ref }}\right) \\
\rho_{v}=\rho_{v}\left(p_{\text {ref }}, T_{\text {ref }}\right)
\end{gathered}
$$

where $p_{\text {ref }}$ and $T_{\text {ref }}$ are prescribed reference pressure and temperature, respectively.

\subsubsection{Void fraction relationship}

Of very numerous works use the mass conservation equation of the vapor phase, eq. (A.2), to compute the cross-sectional average void fraction [24,47]. Despite its apparent appeal, this approach is discarded in the present work. Indeed, not only the right-hand side of eq. (A.2) is at best a cumbersome constitutive relationship and always highly nonlinear, but much worse, when looking for fixed point solutions as base solutions for subsequent linear stability analyses, the related implicit steady-state equation does not ensure the void fraction belongs to its physical domain of definition $(\alpha \in[0,1])$.

Therefore, the alternative way considered in this work is to refer to the mixture mass enthalpy flux (second term on the left hand side of eq. (A.20)), as the relevant closure for the void fraction equation. It somehow translates the thermal equilibrium assumption into the present implicit formalism. However, one first has to derive liquid and vapor enthalpy expressions $\left(h_{l}\right.$ and $h_{v}$ ) dependent on their saturated values $\left(h_{l s}\right.$ and $\left.h_{v s}\right)$ and mixture enthalpy $\left(h_{m}\right)$ in a regularized framework. The derived expressions read as follows:

$$
\begin{aligned}
& h_{l}=h_{l s}+\frac{1}{2}\left[h_{m}-h_{l s}-\sqrt{\left(h_{m}-h_{l s}\right)^{2}+\varepsilon^{2}}\right] \\
& h_{v}=h_{v s}+\frac{1}{2}\left[h_{m}-h_{v s}+\sqrt{\left(h_{m}-h_{v s}\right)^{2}+\varepsilon^{2}}\right]
\end{aligned}
$$

where $\varepsilon$ is the regularization parameter. These regularized liquid and vapor enthalpy relationships are plotted in Fig. 1 versus dimensionless mixture enthalpy for two values of the regularization parameter $\left(\varepsilon=10^{-1}, 10^{-2}\right)$, inside their respective definition domain $\left(h_{l} \leq h_{l s}=0.5\right.$ and $\left.h_{v s}=1.5 \leq h_{v}\right)$. It is noteworthy that the latter value of the regularization parameter $\left(\varepsilon=10^{-2}\right)$ performs quite well in the chosen dimensionless mixture enthalpy framework.

So, one can now easily access to liquid-vapor relative enthalpy $\left(H_{r_{v l}}\right)$ within the phase-change interval $\left(h_{l s} \leq h_{m} \leq h_{v s}\right)$, the only where it has a physical meaning. It reads:

$$
H_{r_{v l}}=h_{v s}-h_{l s}
$$

Substituting then, on the one hand expressions of liquid and vapor velocities (eq. (A.10) and eq. (A.11)) and on the other hand those of regularized liquid and vapor enthalpies (eq. (10) and eq. (11)) into the mixture mass flux enthalpy and equating to its value in eq. (6), one ends up with an implicit void fraction equation as a function of the mixture mass flux, mixture enthalpy, relative liquid-vapor velocity and related thermo-physical fluid properties. It reads: 


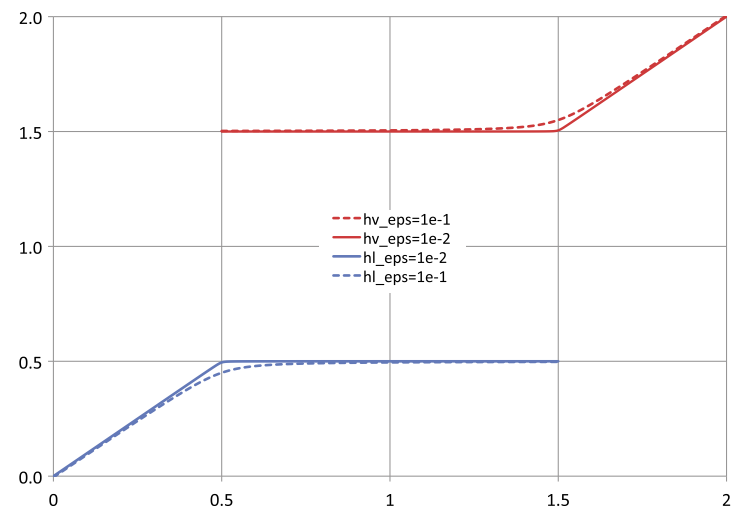

Fig. 1. Dimensionless regularized liquid and vapor enthalpies (blue and red lines, respectively) versus dimensionless mixture enthalpy $\left(\frac{2 h_{m}}{h_{l s}+h_{v_{s}}}\right)$ for $\frac{2\left(h_{v_{s}}-h_{l_{s}}\right)}{h_{l_{s}}+h_{v_{s}}}=1$. Dashed and solid lines refer to regularization parameter $\varepsilon=10^{-1}$ and $10^{-2}$, respectively. (For interpretation of the colors in the figure(s), the reader is referred to the web version of this article.)

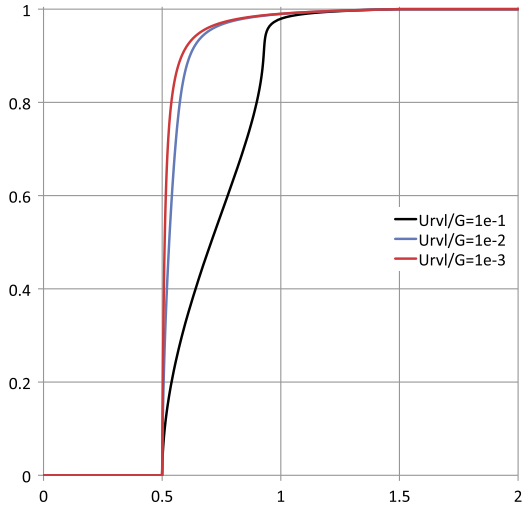

(a) $\rho_{l} / \rho_{v}=100$.

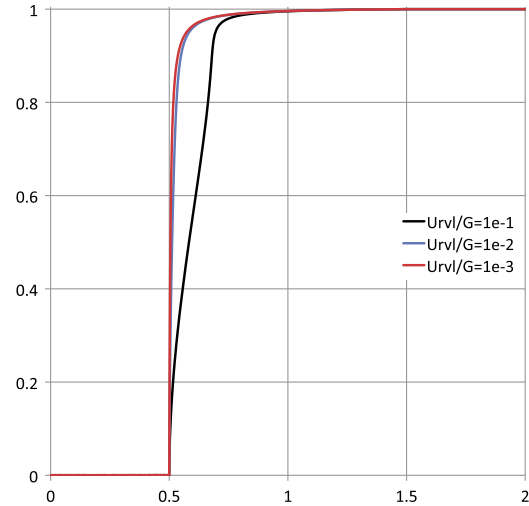

(b) $\rho_{l} / \rho_{v}=250$.

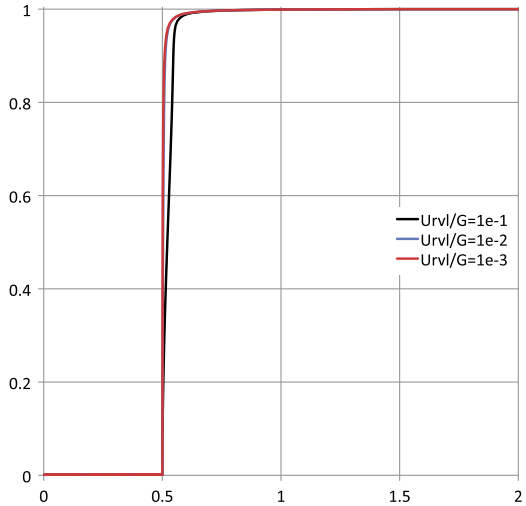

(c) $\rho_{l} / \rho_{v}=1000$.

Fig. 2. Full-range regularized void fraction, eq. (13), versus dimensionless mixture enthalpy $\left(\frac{2 h_{m}}{h_{l_{s}}+h_{v_{s}}}\right)$ for $\frac{2\left(h_{v_{s}}-h_{l_{s}}\right)}{h_{l_{s}}+h_{v_{s}}}=1, \varepsilon=10^{-4}, G=100 \mathrm{~kg} \cdot \mathrm{s}^{-1} \cdot \mathrm{m}^{-2}$, $\rho_{l}=10^{3} \mathrm{~kg} \cdot \mathrm{m}^{-3}$ and various liquid to vapor density ratios and relative velocities, subsequently defined in eq. (15). (a) $\rho_{v}=10 \mathrm{~kg} \cdot \mathrm{m}^{-3} ;$ (b) $\rho_{v}=4 \mathrm{~kg} \cdot \mathrm{m}^{-3}$; (c) $\rho_{v}=1 \mathrm{~kg} \cdot \mathrm{m}^{-3}$.

$$
\begin{aligned}
G\left[(1-\alpha) \rho_{l}\left(h_{m}-h_{l s}+\sqrt{\left(h_{m}-h_{l s}\right)^{2}+\varepsilon^{2}}\right)+\alpha \rho_{v}\left(h_{m}-h_{v s}-\sqrt{\left(h_{m}-h_{v s}\right)^{2}+\varepsilon^{2}}\right)\right] \\
+\alpha(1-\alpha) \rho_{l} \rho_{v} U_{r_{v l}}\left(h_{v s}-h_{l s}-\sqrt{\left(h_{m}-h_{l s}\right)^{2}+\varepsilon^{2}}-\sqrt{\left(h_{m}-h_{v s}\right)^{2}+\varepsilon^{2}}\right)=0
\end{aligned}
$$

This regularized void fraction relationship, eq. (13), is plotted versus dimensionless mixture enthalpy in Fig. 2 for prescribed mass flux and vapor to liquid saturation enthalpy ratio, but various liquid to vapor density ratios and relative velocities. One can note that the higher the liquid to vapor density ratio, the steeper the void fraction evolves just beyond the saturated liquid enthalpy and sharply asymptotes unity. Conversely, the higher liquid-vapor relative velocity, the more void fraction is shifted towards higher mixture enthalpies.

The set of the above three conservation equations (mass eq. (3), momentum eq. (4) and enthalpy eq. (6)) supplemented with void fraction relationship eq. (13) makes up the core of the present one-dimensional model that enables to implicitly solve for their associated four primary variables, namely: $p_{m}, G, h_{m}$ and $\alpha$, respectively. The remaining five variables ( $U_{r_{v l}}$, $\beta, \tau_{l}, \tau_{v}, F_{i_{l v}}$ ) are thus defined as secondary variables, derived as explicit functions of these four primary unknowns.

\subsubsection{Liquid-vapor relative velocity}

The drift-flux model first developed by Zuber \& Findlay [59] in the mid-sixties relies on an empirical constitutive relationship that relates vapor velocity $\left(u_{v}\right)$ to superficial mixture velocity $\left(j=(1-\alpha) u_{l}+\alpha u_{v}\right)$ and drift velocity $\left(v_{D}\right)$, according to the following linear equation:

$$
u_{v}=C_{0} j+v_{D}
$$

where $C_{0}$ is the distribution parameter that accounts for flow patterns in which, liquid and vapor phases are not uniformly distributed in the tube cross-section (e.g., annular flow regime). The empirical constitutive relationship of eq. (14) has been 


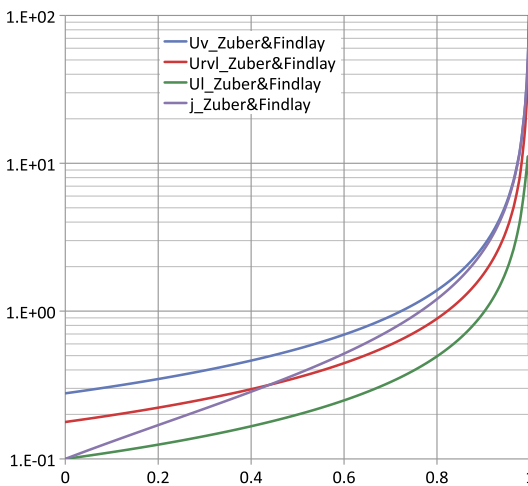

(a) Constant drift velocity.

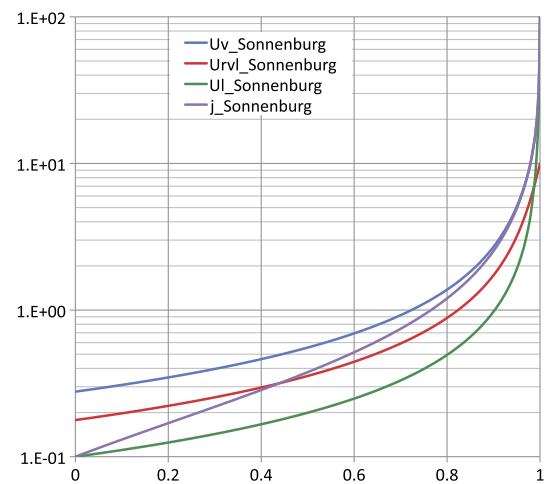

(b) Drift velocity from [50].

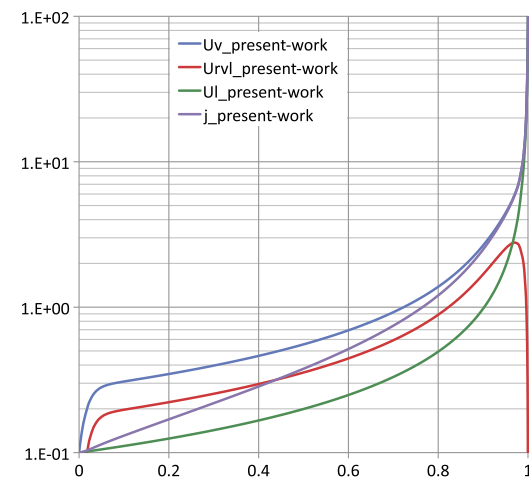

(c) Present drift velocity.

Fig. 3. Liquid, vapor, relative and specific velocities versus void fraction for $G=100 \mathrm{~kg} \cdot \mathrm{s}^{-1} \cdot \mathrm{m}^{-2}, \rho_{l}=10^{3} \mathrm{~kg} \cdot \mathrm{m}^{-3}, \rho_{v}=1 \mathrm{~kg} \cdot \mathrm{m}^{-3}$ and $C_{0}=1$. (a) Constant drift velocity $\left(v_{D}=0.178 \mathrm{~m} . \mathrm{s}^{-1}\right)$; (b) drift velocity from [50]; (c) drift velocity derived in the present work, eq. (B.9). Liquid velocity, eq. (B.2), green line; vapor velocity, eq. (B.3), blue line; relative velocity, eq. (B.1), red line; specific mixture velocity, purple line.

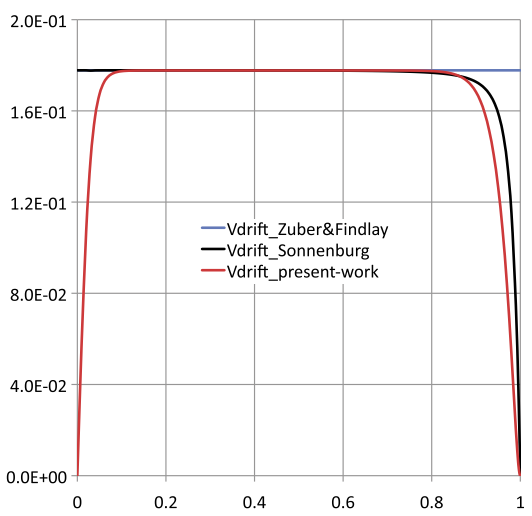

(a) Drift velocity versus void fraction.

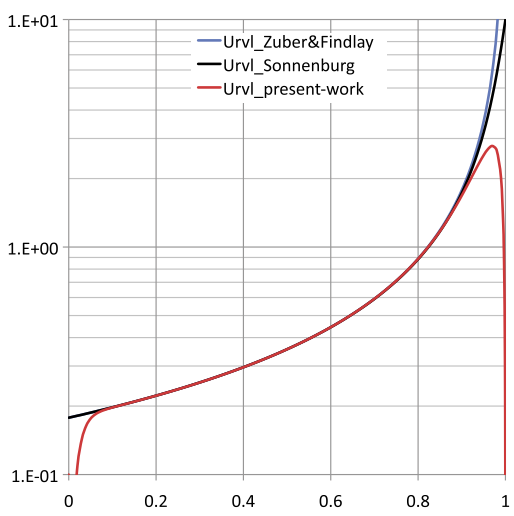

(b) Relative velocity versus void fraction.

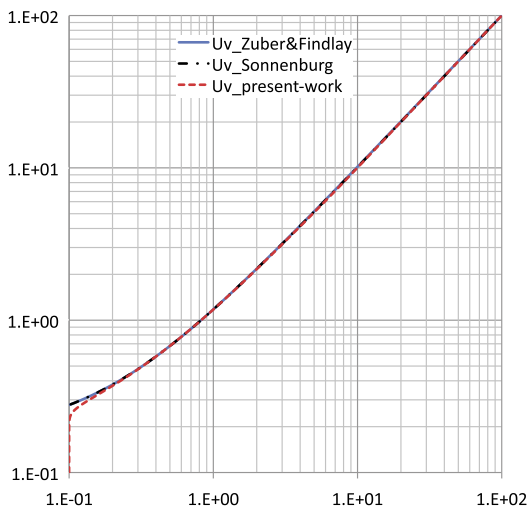

(c) Vapor velocity vs specific mixture velocity

Fig. 4. Comparison of three drift-flux models for $G=100 \mathrm{~kg} \cdot \mathrm{s}^{-1} \cdot \mathrm{m}^{-2}, \rho_{l}=10^{3} \mathrm{~kg} \cdot \mathrm{m}^{-3}, \rho_{v}=1 \mathrm{~kg} \cdot \mathrm{m}^{-3}$ and $C_{0}=1$. Constant drift velocity ( $v_{D}=0.178$ $m . s^{-1}$, blue line), drift velocity from [50] (black line) and present work (red line).

successfully confronted to experiments in many reference works on boiling channel [18,42,47]. To get some insight in how this constitutive relationship acts on the fluid flow, its related velocities have been derived in B.1 and plotted in Fig. 3(a) versus void fraction for constant drift velocity $\left(v_{D}=0.178 \mathrm{~m} . \mathrm{s}^{-1}\right)$ and distribution parameter $\left(C_{0}=1\right), G=100 \mathrm{~kg} . \mathrm{s}^{-1} . \mathrm{m}^{-2}$, $\rho_{l}=10^{3} \mathrm{~kg} \cdot \mathrm{m}^{-3}, \rho_{v}=1 \mathrm{~kg} \cdot \mathrm{m}^{-3}$. With such a constant drift velocity, all velocities except the vapor phase one diverge to infinity as void fraction approaches to unity, which is obviously nonphysical. Therefore, none constant drift velocity can be a candidate for any full-range model, so one should instead look for a constitutive equation in which the drift velocity depends on void fraction as, e.g., in [24,25,50]. Fluid flow velocities corresponding to the drift velocity from [50] are plotted versus void fraction in Fig. 3(b). All velocities are now perfectly bounded, which represents a noticeable improvement with respect to the constant drift velocity model. However, despite various levels of sophistication, none of the quoted boiling channel models simultaneously satisfies all of the present work constraints: full-range implicit constitutive relationship that satisfies conditions of eq. ((B.6)-(B.8)) and that has continuity and derivability properties suitable for our Asymptotic Numerical Method.

Therefore, to overcome these issues we have followed the former approach of Ishii [28] to derive a kinematic constitutive equation that relates the liquid-vapor relative velocity $\left(U_{r_{v l}}\right)$ to mixture mass flux and void fraction, while preserving the global behavior of the original constitutive equation (14). This fully-implicit simplified model is tailored to overcome the weaknesses of previous constitutive equations [50,59]. For the sake of brevity, it is derived in the present paper for the particular case of uniform distribution coefficient $\left(C_{0}=1\right)$. The fluid flow velocities related to the constitutive equation derived in the present work, eq. (B.9), are plotted in Fig. 3(c). One can observe it behaves as expected towards the two single-phase liquid $(\alpha \rightarrow 0)$ and vapor $(\alpha \rightarrow 1)$ limits, respectively, while reproducing faithfully the original constitutive equation in-between.

The drift velocities from the three models (constant, from [50] and present model, eq. (B.9)) are plotted versus void fraction in Fig. 4(a). They coincide over the main part of the void fraction range except at both ends, where the drift velocity introduced in the present work tends to zero faster than that from [50] for void fraction approaching to one and is 


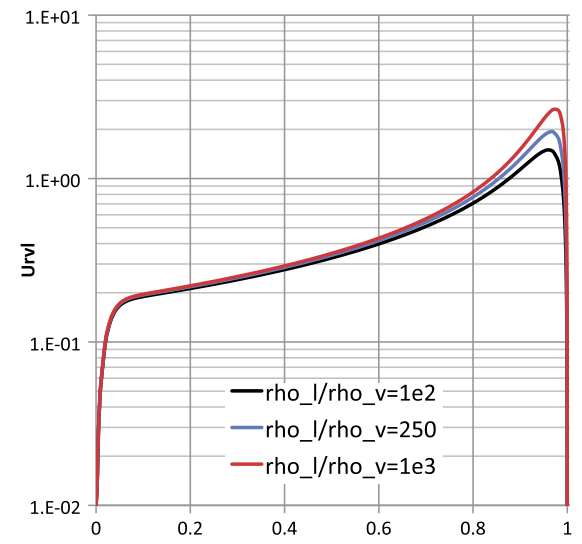

(a) Relative liquid-vapor velocity.

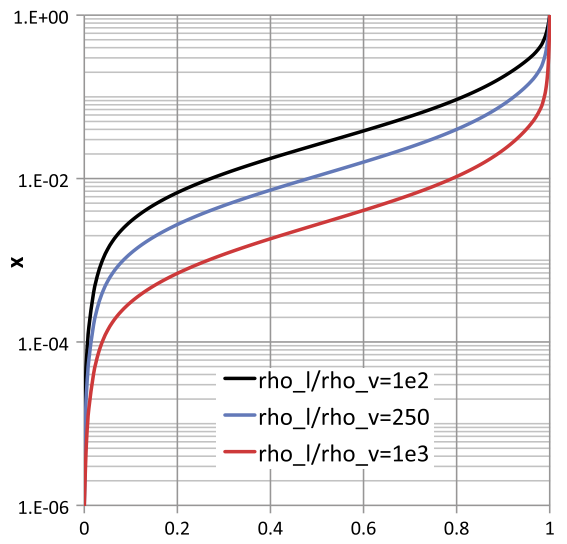

(b) Vapor quality.

Fig. 5. Relative liquid-vapor velocity (eq. (15)) and vapor quality (eq. (16)) versus void fraction for $d=10^{-2} \mathrm{~m}, \mathrm{G}=100 \mathrm{~kg} \cdot \mathrm{s}^{-1} \cdot \mathrm{m}^{-2}, \rho_{l}=10^{3} \mathrm{~kg} \cdot \mathrm{m}^{-3}$, $C_{0}=1$ and various liquid to vapor density ratios $\left(\rho_{v}=10,4,1 \mathrm{~kg} . \mathrm{m}^{-3}\right)$.

the only one that goes to zero for $\alpha$ tending to zero in order to satisfy eq. (B.6). Substituting back the proposed constitutive relationship of eq. (B.9) into the liquid-vapor relative velocity expression of eq. (B.1), performing then some tedious algebra (expanding, developing into series, simplifying and identifying leading order terms) thanks to the Mathematica [54] symbolic algebra software, the full-range analytical expression of the liquid-vapor relative velocity reads:

$$
U_{r_{v l}}=v_{D_{0}} \frac{\tanh \left(a_{u} \alpha\right)\left(1-\alpha^{c_{u}}\right)}{1-\alpha\left(1-b_{u}\right)}
$$

where $v_{D_{0}}=\frac{9}{16} \sqrt{\frac{\operatorname{gd}\left(\rho_{l}-\rho_{v}\right)}{\rho_{l}}}$ is the drift velocity in the limit of $\alpha \rightarrow 0$ from [50] and the three constants have been identified to: $a_{u}=32, b_{u}=\frac{9}{16} \sqrt{\frac{\rho_{v}}{\rho_{l}}}$ and $c_{u}=40$. The liquid-vapor relative velocities from the three models (constant drift velocity, from [50] and present model, eq. (15)) are plotted versus void fraction in Fig. 4(b) for the considered case and the same comment as for the drift velocity plot also holds. Finally, vapor velocities from the three models are plotted versus specific mixture velocity in Fig. 4(c) and one can notice the very good agreement to the original constitutive relationship [59] of eq. (14) over most of the void fraction range, except close to the pure liquid region $(\alpha \rightarrow 0)$ where the present model departs from the two others as its vapor velocity tends to liquid one in order to satisfy continuity conditions of eq. ((B.4)-(B.5)). The influence of liquid to vapor density ratio is depicted in Fig. 5(a) and it is noteworthy that the higher this ratio, the stronger is the vapor-liquid relative velocity as its maximum evolves as $\sqrt{g d\left(\frac{\rho_{l}}{\rho_{v}}-1\right)}$.

Finally, vapor quality can be expressed with respect to our primary variables of the problem (mixture mass flux, void fraction) and relative liquid-vapor velocity, in the following way:

$$
x=\frac{\alpha \rho_{v} u_{v}}{G}=\frac{\alpha \rho_{v}\left[G+(1-\alpha) \rho_{l} U_{r_{v l}}\right]}{G\left[(1-\alpha) \rho_{l}+\alpha \rho_{v}\right]}
$$

Vapor quality, eq. (16), is plotted versus void fraction in Fig. 5(b) for three liquid to vapor density ratios. It turns out that the higher the density ratio, the smaller is vapor quality for a given void fraction.

\subsubsection{Two-phase frictional pressure drop}

For single-phase fluid flows in tubes the frictional stress is related to friction coefficient and flow kinetic energy as follows:

$$
\tau_{i}=\frac{f_{i}}{4} \frac{G^{2}}{2 \rho_{i}}
$$

where $f_{i}$ is the Darcy-Weisbach friction coefficient of the $i$-fluid phase (subscript $i=l, v$ ). It can be defined by numerous correlations among them the Churchill constitutive law [7], that reads:

$$
f_{i}=8\left\{\left(\frac{8}{R e_{i}}\right)^{12}+\left[\left(-2.457 \ln \left[\left(\frac{7}{R e_{i}}\right)^{0.9}+0.27 \frac{\epsilon_{r}}{d}\right]\right)^{16}+\left(\frac{37530}{R e_{i}}\right)^{16}\right]^{-3 / 2}\right\}^{1 / 12}
$$




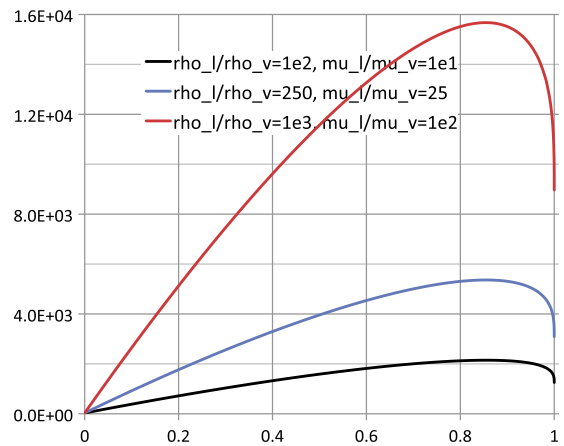

(a) Two-phase frictional pressure gradient vs $x$.

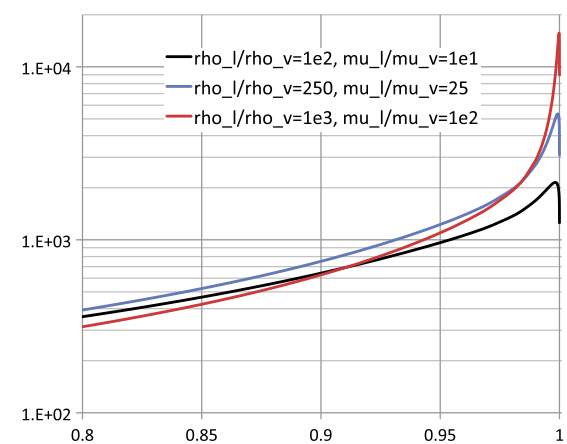

(b) Two-phase frictional pressure gradient vs $\alpha$.

Fig. 6. Two-phase frictional pressure gradient, eq. (19) for $d=10^{-2} \mathrm{~m}, \mathrm{G}=100 \mathrm{~kg} \cdot \mathrm{s}^{-1} \cdot \mathrm{m}^{-2}, \rho_{l}=10^{3} \mathrm{~kg} \cdot \mathrm{m}^{-3}, \mu_{l}=10^{-3}$ Pa. $\mathrm{s}^{-1}$ and various liquid to vapor density and dynamic viscosity ratios $\left(\rho_{v}=10,4,1 \mathrm{~kg} \cdot \mathrm{m}^{-3} ; \mu_{v}=10^{-4}, 410^{-5}, 10^{-5} \mathrm{~Pa} \cdot \mathrm{s}^{-1}\right)$. (a) versus vapor quality; (b) versus void fraction.

where $R e_{i}=\frac{G d}{\mu_{i}}$ is the related Reynolds number ( $\mu_{i}$ is the dynamical viscosity of the $i$-fluid phase) and $\epsilon_{r}$ is the tube roughness. This correlation performs quite well for single-phase tube flows at all flow regimes (laminar, transitional and turbulent) as it recovers the Hagen-Poiseuille friction coefficient $\left(f_{i}=\frac{64}{R e_{i}}\right)$ and the Blasius one $\left(f_{i}=0.3164 R e_{i}^{-\frac{1}{4}}\right)$ in laminar and fully turbulent regimes, respectively.

For two-phase flows, very few correlations are able to predict their frictional pressure drop for all flow regimes and flow patterns. However, the Muller-Steinhagen constitutive relationship [37] chosen in this work is one of the simplest and it performs surprisingly among the best over a broad range of flow configurations [51,57]. It enables to relate the two-phase frictional pressure gradient to vapor quality and individual contributions of liquid and vapor phases flowing alone in the tube. Thanks to this relationship the two-phase frictional pressure gradient that appears in the momentum conservation equation of the mixture (second term on right hand side of eq. (4)) can be approximated as follows:

$$
\left(\frac{\partial p_{m}}{\partial z}\right)_{f r i c}=\left[(1-\beta) \tau_{l}+\beta \tau_{v}\right] \frac{4}{d} \approx\left\{\left[\frac{f_{l}}{\rho_{l}}+2\left(\frac{f_{v}}{\rho_{v}}-\frac{f_{l}}{\rho_{l}}\right) x\right](1-x)^{\frac{1}{c}}+\frac{f_{v}}{\rho_{v}} x^{c}\right\} \frac{G^{2}}{2 d}=f_{m} \frac{G^{2}}{2 d}
$$

where $x$ is the vapor quality and the $c$ exponent is a constant (its value, $c=3$, results from fits on thousand of experimental data points [37]) and $f_{m}$ is the resulting mixture friction coefficient. This correlation works fine provided $\frac{f_{v}}{\rho_{v}}-\frac{f_{l}}{\rho_{l}}>0$, that is to say, when the kinematic viscosity of vapor phase is greater than liquid one $\left(\frac{\mu_{v}}{\rho_{v}}>\frac{\mu_{l}}{\rho_{l}}\right)$, condition which is usually satisfied for low to moderate viscosity liquids. The two-phase frictional pressure gradient (eq. (19)) is plotted versus vapor quality and void fraction in Figs. 6(a) and 6(b), respectively, for three liquid to vapor density ratios and dynamic viscosity ones. One can observe that it first increases up to its maximum value that takes place at roughly $x=0.85$ and then it steeply decreases to the pure vapor value.

\subsubsection{Mechanical power of phase interaction force discarded}

In the present one-dimensional model neither the topology of the liquid-vapor interface nor its dynamics are by no way accessible to any implicit computation. However, the mechanical power of this interaction term that acts at the liquid-vapor interface can be legitimately assumed negligible with respect to the leading terms (heating power, mixture mass enthalpy flux, etc.) appearing in the enthalpy conservation equation. Consequently, in what follows the related term $\left(F_{i_{l v}} U_{r_{v l}}\right)$ is always neglected in eq. (6).

\subsection{Dimensionless set of governing equations}

Setting the tube length as length scale, $\tilde{L}=l$, then related dimensionless length quantities read: $\hat{z}=z / \tilde{L}, \hat{d}=d / \tilde{L}$; setting then reference density and mass flux, respectively denoted $\tilde{\rho}$ and $\tilde{G}$, then the reference time and pressure read: $\tilde{t}=\tilde{\rho} \tilde{L} / \tilde{G}$ and $\tilde{p}=\tilde{G}^{2} / \tilde{\rho}$, respectively. Finally, the reference enthalpy is set to $\tilde{h}$. Therefore, the present one-dimensional full-range liquid-vapor two-phase flow model is governed by the set of four implicit equations related to the four primary dimensionless unknowns, namely $\hat{p}_{m}=p_{m} / \tilde{p}, \hat{G}=G / \tilde{G}, \hat{h}_{m}=h_{m} / \tilde{h}$ and $\alpha$, that read as follows:

$$
\begin{aligned}
& \frac{\partial \hat{\rho}_{m}}{\partial \hat{t}}=-\frac{\partial \hat{G}}{\partial \hat{z}} \\
& \frac{\partial \hat{G}}{\partial \hat{t}}=-\frac{\partial}{\partial \hat{z}}\left[\frac{\hat{G}^{2}}{\hat{\rho}_{m}}+\alpha(1-\alpha) \frac{\hat{\rho}_{l} \hat{\rho}_{v}}{\hat{\rho}_{m}} \frac{\tilde{\rho}^{2} U_{r_{v l}}^{2}}{\tilde{G}^{2}}\right]-\frac{\partial \hat{p}_{m}}{\partial \hat{z}}-\hat{f}_{m} \frac{\hat{G}^{2}}{2 \hat{d}}-\frac{\tilde{\rho}^{2} \tilde{L}}{\tilde{G}^{2}} \hat{\rho}_{m} g
\end{aligned}
$$




$$
\begin{aligned}
\frac{\partial}{\partial \hat{t}}\left(\hat{\rho}_{m} \hat{h}_{m}-\frac{\tilde{G}^{2}}{\tilde{\rho}^{2} \tilde{h}} \hat{p}_{m}\right)= & \left.-\frac{\partial}{\partial \hat{z}} \hat{G} \hat{h}_{m}+\alpha(1-\alpha) \frac{\hat{\rho}_{l} \hat{\rho}_{v}}{\hat{\rho}_{m}} \frac{\tilde{\rho} U_{r_{v l}} H_{r_{v l}}}{\tilde{G} \tilde{h}}+\frac{1}{\hat{\rho}_{m}} \frac{\tilde{G}^{2}}{\tilde{\rho}^{2} \tilde{h}} \hat{G}+\alpha(1-\alpha)\left(\hat{\rho}_{l}-\hat{\rho}_{v}\right) \frac{\tilde{\rho} U_{r_{v l}}}{\tilde{G}}\right] \frac{\partial \hat{p}_{m}}{\partial \hat{z}} \\
& +\frac{4 q_{w}}{\tilde{G} \tilde{h} \hat{d}} \\
0= & \hat{G}\left[(1-\alpha) \hat{\rho}_{l}\left(\hat{h}_{m}-\hat{h}_{l s}+\sqrt{\left(\hat{h}_{m}-\hat{h}_{l s}\right)^{2}+\varepsilon^{2}}\right)+\alpha \hat{\rho}_{v}\left(\hat{h}_{m}-\hat{h}_{v s}-\sqrt{\left(\hat{h}_{m}-\hat{h}_{v s}\right)^{2}+\varepsilon^{2}}\right)\right] \\
& +\alpha(1-\alpha) \hat{\rho}_{l} \hat{\rho}_{v} \frac{\tilde{\rho} U_{r_{v l}}}{\tilde{G}}\left(\hat{h}_{v s}-\hat{h}_{l s}-\sqrt{\left(\hat{h}_{m}-\hat{h}_{l s}\right)^{2}+\varepsilon^{2}}-\sqrt{\left(\hat{h}_{m}-\hat{h}_{v s}\right)^{2}+\varepsilon^{2}}\right)
\end{aligned}
$$

\section{Numerical model to compute bifurcation diagrams}

Once the set of governing equations has been closed and is solvable one has to select a numerical strategy to accurately and efficiently compute its relevant solutions. Furthermore, one also wants to determine for which range of control parameters the system is either stable or unstable, as qualitative and quantitative changes in its behavior may occur. The methodological strategy used in this paper follows the Dynamical System Theory $[19,27,32]$ in a way that one first finds out fixed point solutions of the governing equations, then select some relevant ones as base-states at which linear stability analyses are performed. The stability of the system changes at critical values of control parameters associated with, e.g., turning points, steady-state or Hopf bifurcation points, which unfortunately coincide to singular solution of the governing equations $[14,29]$. Therefore, specialized algorithms should be used to accurately compute them $[10,16,17,48,49]$.

The originality of the present work in the framework of boiling fluid flows stands in the way one efficiently computes branches of steady-state solutions. It results from the combination of accurate discretization of their governing partial differential equations and efficient solution of the resulting highly nonlinear algebraic system, thanks to a powerful continuation algorithm based on the Asymptotic Numerical Method [11-13]. So, this section presents its main numerical stages and methods, derived in the framework of the present full-range boiling flow model.

\subsection{Spatial discretization of first-order derivatives}

As just discussed above, one first seeks for fixed points of the set of governing equations, that is to say setting to zero the time derivatives on left-hand sides of eq. (20a)-(20c). This results in a set of nonlinear first-order partial differential equations, which transform into a set of nonlinear algebraic equations after spatial discretization of their first-order derivatives. In such a one-dimensional problem, a simple and accurate way to proceed is to perform spatial discretizations with finite difference schemes. However, mass and momentum conservation equations, eq. ((20a)-(20b)), are linked to one another by density and mass flux, meanwhile the incompressibility condition is assumed to hold, it results that density variations are no longer related to any pressure ones. Therefore, a pressure equation is required such that solutions of the momentum equation be mass-consistent, along with a specific velocity-pressure coupling in order to end-up in a mass consistent stable scheme. So, to overcome these issues and to prevent from any odd-even decoupling or checkerboard pattern for pressure, staggered grids for mass flux and pressure are introduced following the original velocity-pressure coupling scheme first suggested in [23], see Fig. 7.

Let $Q$ be any of the collocated primary variables $\left(G, h_{m}, \alpha\right)$, whose $n$ evenly distributed discrete values along the one dimensional computational domain are located at $Q_{i}(1 \leq i \leq n)$ points, see Fig. 7. Their first-order spatial derivative in momentum or enthalpy conservation equations, eq. ((20b), (20c)), are discretized at every collocated grid point with the 6th order finite difference scheme of eq. (C.1). Furthermore, it is noteworthy to mention that in the present staggered discretization scheme, the $n-1$ discrete pressure unknowns are evenly located at the middle point to their adjacent collocated mates, meanwhile the two pressure boundary conditions $\left(p_{0}\right.$ and $\left.p_{L}\right)$ are set at the two computational domain ends $(\hat{z}=0$ and $\hat{z}=\hat{l}$ ), respectively, to satisfy the actual imposed pressure drop, cf. Fig. 7 . According to the chosen staggered grids the first-order derivative of mixture pressure in the momentum equation, eq. (20b), is computed at every collocated grid point with the 5th order finite difference scheme of eq. (C.2). Finally, the first-order derivative of the mixture mass flux in the mass conservation equation, eq. (20a), is computed at every staggered grid point with the staggered 5th order finite difference scheme of eq. (C.3). To target optimal accuracy all these discretization operators use centered stencils, except in the vicinity of the two computational domain ends where the resulting schemes are forward or backward finite difference stencils, respectively.

\subsection{Continuation algorithm to compute fixed point solutions}

Once the set of nonlinear algebraic equations is at disposal, one wants to efficiently compute its fixed point solutions by continuation or path-following algorithms $[2,29]$. They aim at mapping the parameter space by computing branches of 


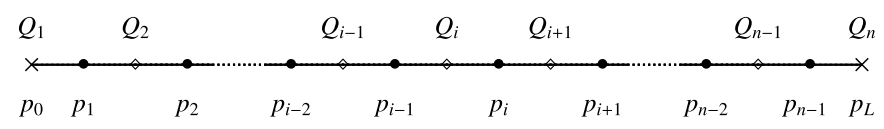

Fig. 7. One-dimensional computational domain of the vertical heated tube (rotated $90^{\circ}$ clockwise for display purpose) and its evenly distributed staggered grids of primary variable unknowns: collocated ones $\left(Q_{i}=G_{i}, h_{m i}, \alpha_{i}\right)$ and staggered ones $\left(p_{i}\right)$.

solutions for a given range of control parameters, along with critical values and their corresponding singular solution. Their founding principle is based on the Implicit Function Theorem [29], which basically states that from a given starting-point solution, provided the jacobian matrix associated with the functional at this point is full rank (regular point), then it exists in its vicinity a branch of solutions that passes throught that point. On the other hand, more complex algebra have to be performed to compute emanating branch(es) from singular points, i.e., steady-state bifurcation points, but specialized algorithms are now well established [13,27,29].

Among continuation algorithms, first-order predictor-corrector ones with pseudo-arc-length parameterization have been widely used for decades [15-17,29,48,49]. Nevertheless, their step-length adaptivity may be sometimes in trouble in the vicinity of bifurcation points, resulting in weak computational efficiency and even worse as possible lack of convergence. An alternative way to first-order predictor algorithms stands in high-order predictors that have been introduced in the Asymptotic Numerical Method (ANM) [11-13]. Its implementation in the frame of the present full-range two-phase flow model involves two main components: a continuation algorithm based on high-order Taylor series expansion and Automatic Differentiation (AD) procedures to efficiently compute their numerous coefficients associated with every nonlinear terms involved in the governing equations. These two parts are in turn briefly presented below.

\subsubsection{Asymptotic Numerical Method}

Unlike first-order predictor-corrector algorithms one of the key features of the Asymptotic Numerical Method is that it does not involve any a priori user-prescribed incremental loading to drive the continuation process. Indeed, it rather translates the latter into an implicit parametric loading problem in such a way that the control parameter enters the algorithm as an unknown loading coefficient. Furthermore, it advantageously combines high-order Taylor series expansion and parameterization strategy resulting in a general and efficient nonlinear solution method. Its intrinsic features enables to transform the initial set of nonlinear algebraic equations into a series of linear sets, sharing the same tangent operator, which basically provides an overall high computational efficiency to the method. In the ANM step-length adaptivity is intimately related with the radius of convergence of the series, so it automatically self-adapts to any local nonlinear changes. Finally, taking advantage of Van Dyke's pioneer works on power series analysis [52] the computational efficiency of the ANM algorithm has been nicely improved at steady bifurcation points and their emanating branches [13].

To briefly present the main features of ANM, let us first introduce some generic notations. Let $R(u, \lambda)=0$ be the functional associated with the algebraic system of $n$ nonlinear smooth equations. The latter results from the spatial discretization of the set of steady-state governing equations. $u \in \mathbb{R}^{n}$ is the vector of discretized state variable unknowns ( $G, p$, $h_{m}$ and $\alpha$ values at their respective grid points) and $\lambda \in \mathbb{R}$ the chosen scalar control parameter. The extended state vector $U=\left[\begin{array}{l}u \\ \lambda\end{array}\right] \in \mathbb{R}^{n+1}$ is in turn introduced for compactness as it contains the continuation parameter $\lambda$ in addition to state variables, so that the equilibrium system now reads:

$$
R(U)=0
$$

Generic solutions of eq. (21) are branches of solutions, which are represented in ANM continuation algorithms by the power series expansion of eq. (22):

$$
U(a)=U_{0}+a U_{1}+a^{2} U_{2}+\cdots+a^{m} U_{m}
$$

where $m$ is the truncate order of the power series expansion and $a$ the path parameter defined in the parameterization equation (23) as the classical pseudo arc-length [11,12]:

$$
a=\left[U(a)-U_{0}\right]^{T} \cdot U_{1}
$$

Substituting the power series expansion of eq. (22) into the original algebraic set of nonlinear equations (21), equating then like powers of $a$, it results a set of linear algebraic systems, that share a unique tangent operator (the jacobian matrix) and have recursive right hand sides. The latter depends on the solution vectors from previous series expansion orders, therefore, one has to solve sequentially at every order of the Taylor series expansion for the unknowns of these recursive linear algebraic systems to provide the sought nonlinear solution in the vicinity of a known starting point.

However, as any Taylor series expansion has generally only a finite radius of convergence, one has to compute the maximum value of the path parameter to not exceed a user-defined maximum departure from the solution curve within the ANM predictor step. For that purpose we make use of a reliable criterion to evaluate the maximum path parameter value $a_{\max }$. It is built on the ratio between the first term of the series to the last one, times a user-prescribed accuracy constant $\delta$ [11]: 


$$
a_{\max }=\left(\delta \frac{\left\|\vec{U}_{1}\right\|}{\left\|\vec{U}_{m}\right\|}\right)^{\frac{1}{m-1}}
$$

In that way, the ANM algorithm enables to compute a piecewise semi-analytical representation of the whole path in a step-by-step procedure until the parameter range of interest has been spanned. Moreover, as the ANM predictor step could produce a guess solution slightly departing from the sought solution curve, the classical Newton-Raphson algorithm is implemented as a subsequent corrector step, when needed.

\subsubsection{Automatic Differentiation}

On the one hand, constitutive relationships or closures supplemented to the conservation equations have been carefully designed to be appropriately continuous and differentiable in the framework of Taylor series expansion involved in ANM. On the other hand, the overall computational efficiency of ANM highly depends on computational costs associated with recurrence formulas involved in the nonlinear terms appearing in the right hand side of linear algebraic systems to be solved at every order of the asymptotic expansion. Since the present set of governing equations involve numerous highly nonlinear terms it has been a good opportunity to resort to Automatic Differentiation in the framework of the Asymptotic Numerical Method, thanks to its object-oriented implementation in the Diamanlab software [5,6].

Automatic Differentiation is used in the Diamanlab software to compute first-order derivatives involved in the jacobian matrix once per ANM predictor step and high-order derivatives appearing in recursive right hand sides at every asymptotic expansion order. Its basic principle is to make a systematic use of complex function decomposition into elemental differential ones and then apply differentiation chain-rule to composition of those elemental functions. The computation of Taylor series coefficients of elementary functions and arithmetic operations (sum, product, etc.) is performed thanks to recurrence formulas such as the famous Leibniz formula for a product [22] and the Faà di Bruno's generalization for the chain-rule to higher-order derivatives [56]. The implementation in the Diamanlab software [5,6] relies on operator overloading as the vehicle of attaching higher-order derivative computations to arithmetic operators and intrinsic functions provided by the programming language.

\subsection{Linear stability analysis}

Once branches of fixed point solutions have been computed, linear stability analyses (LSA) can be performed on some of these steady-state solutions that can interestingly belong to the highly nonlinear regime. In this goal, one follows the Dynamical System Theory [19,27,32], because although it has been developed for Ordinary Differential Equations (ODEs), the needed theoretical background and formalisms can be transposed and adapted to Partial Differential Equations (PDEs) involved in the present set of governing equations.

Let $u^{s}(z, \lambda)$ be one of the steady-state solutions previously computed for a particular value of the control parameter, it is referred to as a base-state. The most simple way to perform a linear stability analysis is to superimpose to this base-state an infinitely small amplitude perturbation. Then, a classical method to transform the initial-value PDEs problem into a corresponding eigenvalue one is to introduce the normal mode approach, in which the space-time variable separation of the perturbation field is of the form $u^{p}(z, t)=\phi(z) e^{\sigma t}, \phi(z)$ being the spatial mode and $\sigma$ the temporal growth or decay rate. In that way, the linear stability analysis produces a linearization of the evolution equations around this steady-state solution. For the present governing equations this procedure leads to a generalized eigenvalue problem that reads as follows:

$$
[\mathbf{J}]\{\phi\}=\sigma[\mathbf{M}]\{\phi\}
$$

where $[\mathbf{J}]=\left[\frac{\partial R(u, \lambda)}{\partial u}\right]$ is the jacobian matrix at steady-state solution point $u^{s}(z, \lambda)$ and $[\mathbf{M}]$ is the mass matrix at that point (i.e., coefficient matrix of the time derivative terms). The eigenvalues $\sigma$ and their associated eigenvectors $\phi$ can be complex numbers and vectors, respectively. So, if the real part of the rightmost eigenvalue of eq. (25) is negative (positive), the system is linearly stable (unstable), else it is marginally or neutrally stable.

The main issue arising from the generalized eigenvalue problem of eq. (25) is the mass matrix associated with the discretization of dynamical governing equations is singular, so there are as many infinite eigenvalues as singular equations in the system. The first source of singularity is associated with the incompressibility constraint that assumes density does not depend on pressure. This is a classical issue which has been broadly addressed in the LSA literature of incompressible fluid flows $[8,9,35]$. However, in the present problem where liquid-vapor phase-change is involved, incompressibility does not lead to divergence-free response to dynamical perturbations as density could vary up to three orders of magnitude (for water-steam phase-change at atmospheric pressure, $\frac{\rho_{l}}{\rho_{v}}=O\left(10^{3}\right)$ ). So, most of the numerous algorithms devoted to divergence-free problems associated with constant density assumption are no longer appropriate to solve eq. (25). However, they inspired us in the way to undertake the second singularity of the problem, which is associated with the fully implicit time-independent closure equation of the void fraction, eq. (20d). 


$$
[\mathbf{J}]=\left(\begin{array}{cccc}
J_{G G} & J_{G p} & 0 & J_{G \alpha} \\
J_{p G} & 0 & 0 & 0 \\
J_{h G} & J_{h p} & J_{h h} & J_{h \alpha} \\
J_{\alpha G} & 0 & J_{\alpha h} & J_{\alpha \alpha}
\end{array}\right) ; \quad[\mathbf{M}]=\left(\begin{array}{cccc}
M_{G G} & 0 & 0 & 0 \\
0 & 0 & 0 & M_{p \alpha} \\
0 & M_{h p} & M_{h h} & M_{h \alpha} \\
0 & 0 & 0 & 0
\end{array}\right)
$$

Indeed, taking advantage of the block structure of the algebraic system, cf. eq. (26), resulting from the discretization of the set of linearized perturbation equations, one can perform a static condensation of the algebraic system to eliminate the $n$ perturbation equations associated with void fraction unknowns. The corresponding matrices resulting from this static condensation read, cf. eq. (27):

$$
\left[\mathbf{J}_{s c}\right]=\left(\begin{array}{ccc}
J_{G G}-J_{G \alpha} J_{\alpha \alpha}^{-1} J_{\alpha G} & J_{G p} & -J_{G \alpha} J_{\alpha \alpha}^{-1} J_{\alpha h} \\
J_{p G} & 0 & 0 \\
J_{h G}-J_{h \alpha} J_{\alpha \alpha}^{-1} J_{\alpha G} & J_{h p} & J_{h h}-J_{h \alpha} J_{\alpha \alpha}^{-1} J_{\alpha h}
\end{array}\right) ;\left[\mathbf{M}_{s c}\right]=\left(\begin{array}{ccc}
M_{G G} & 0 & 0 \\
-M_{p \alpha} J_{\alpha \alpha}^{-1} J_{\alpha G} & 0 & -M_{p \alpha} J_{\alpha \alpha}^{-1} J_{\alpha h} \\
-M_{h \alpha} J_{\alpha \alpha}^{-1} J_{\alpha G} & M_{h p} & M_{h h}-M_{h \alpha} J_{\alpha \alpha}^{-1} J_{\alpha h}
\end{array}\right)
$$

The singularity of the resulting generalized eigenvalue problem is reduced, but $\left[\mathbf{M}_{s c}\right]$ is still singular. Indeed, it remains $n-1$ infinite eigenvalues associated with pressure unknowns out of $3 n-2$ discrete equations, $n$ being the number of grid points. The direct matrix factorization of the reduced size system is reasonably affordable for the present one-dimensional model as its cost evolves as $O\left((3 n-2)^{3}\right)$. Therefore, the present generalized eigenvalue problem with singular mass matrix can be accurately solved thanks to the Jacobi-Davidson $Q Z$ factorization algorithm $[20,36,45,53]$. Let $\left[\mathbf{J}_{s c}^{U T}\right]$ and $\left[\mathbf{M}_{s c}^{U T}\right]$ be the upper triangular matrices resulting from the $Q Z$ factorization of $\left[\mathbf{J}_{s c}\right]$ and $\left[\mathbf{M}_{s c}\right]$, respectively, then $\{\beta\}=\operatorname{diag}\left[\mathbf{J}_{s c}^{U T}\right]$ and $\{\delta\}=\operatorname{diag}\left[\mathbf{M}_{s c}^{U T}\right]$ are the generalized eigenvalues that satisfy:

$$
\left[\mathbf{J}_{s c}\right]\left\{\phi_{s c}\right\} \cdot\{\delta\}=\left[\mathbf{M}_{s c}\right]\left\{\phi_{s c}\right\} \cdot\{\beta\}
$$

Finally, the $2 n-1$ relevant eigenvalues (that are not infinite) are conditionally computed according to the following rule: for $1 \leq i \leq 3 n-2$; if $\delta_{i}>\varepsilon$ or $0.1 \leq\left|\beta_{i} \delta_{i}\right| / \varepsilon^{2} \leq 10$, then $\sigma_{s c_{i}}=\beta_{i} / \delta_{i}$ (with $\varepsilon$ being a user-defined tolerance). This procedure has been satisfactorily validated against classical Shift-and-Invert and Generalized Cayley spectral transform methods [8,10, 35].

\section{Numerical assessments}

Several numerical analyses have been performed on representative test cases to assess computational accuracy and efficiency of the present implementation. Let us consider a natural circulation flows into an open heated tube (length $l=10 \mathrm{~d}$, diameter $d=10^{-2} \mathrm{~m}$ ) immersed into a liquid water bath of infinite extension at atmospheric pressure. Continuation in this sub-cooled natural circulation configuration is performed starting from a base-state defined at zero heating power by sub-cooled liquid water $\left(\hat{h}_{m}^{0}<\hat{h}_{l s}\right)$ at rest in hydro-static equilibrium. A constant heat flux is supplied at tube wall over the first eighty percents of its length $\left(l_{q} / l=0.8\right)$, while the remaining twenty percents are assumed adiabatic. Natural circulation into the tube is computed by taking the heating power as continuation parameter in such a way one travels along the branch of solutions for which the mixture enthalpy of the fluid monotonically increases. The present continuation procedure consists in computing steady-state solutions versus tube heating power. This section presents at first a spatial convergence analysis and secondly a sensitivity analysis with respect to the main numerical parameter of the model.

\subsection{Spatial convergence analysis}

A spatial convergence analysis is performed on branches of steady-state solutions and bifurcation points (Hopf and turning point) where the linear stability of base-state changes. Approximate solutions have been computed for a value of the regularization parameter $\varepsilon=10^{-3}$ on four successively refined grids (npts $=201,401,801$ and 1601 points) to achieve reliable spatial convergence, so that Richardson extrapolation (RE) can be performed. This extrapolation technique not only enables to evaluate effective convergence order for any actual problem of interest, but also and more importantly, to improve the accuracy of discrete solutions when used in the asymptotic range $[38,39,43,44]$.

\subsubsection{Branches of steady-state solutions}

Branches of steady-state solutions are plotted in Fig. 8 versus dimensionless heating power per cross section area $\left(\hat{\lambda}=4 \frac{q_{w}}{\tilde{q}} \frac{l}{d}\right)$ as continuation parameter for the four spatial discretizations considered. In this sub-cooled natural circulation configuration continuation curves exhibit two turning points. In a first stage, buoyancy increases faster than resisting forces (wall friction and phase-change acceleration) so that mass flux increases along with heating power. Then, a first turning point occurs along the continuation for its maximum heating power $\left(\hat{\lambda}_{T P_{1}} \approx 0.4932\right)$ and maximum mass flux $\left(\hat{G}_{T P_{1}} \approx 0.5172\right)$, leading to a mixture enthalpy at tube outlet $\left(\hat{h}_{m}(\hat{l})_{T P_{1}} \approx 0.4009\right)$ just above its liquid saturation value $\left(\hat{h}_{l s}=0.4\right)$ and a moderate void fraction at tube outlet $\left(\alpha(\hat{l})_{T P_{1}} \approx 0.3420\right)$. Beyond this stage, two-phase frictional pressure drop and phase-change acceleration increase faster than buoyancy so that the mixture mass flux decreases. It results that 


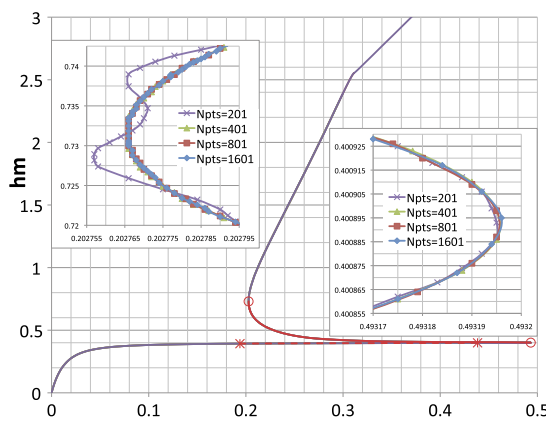

(a) Mixture enthalpy at tube outlet $(\hat{z}=1)$

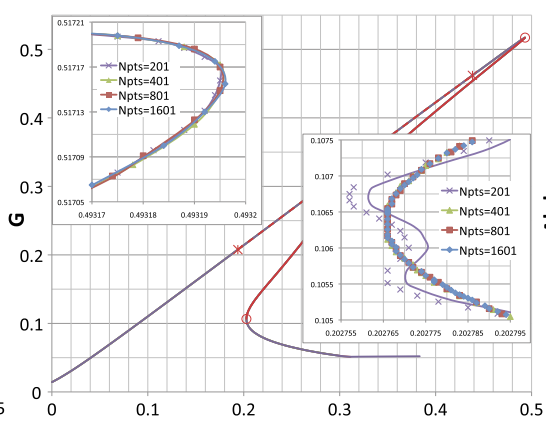

(b) Mixture mass flux across tube.

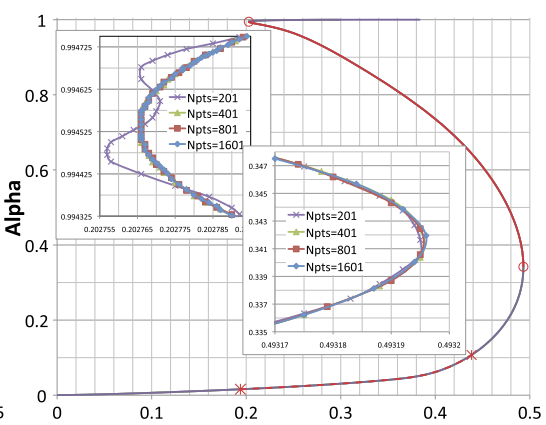

(c) Void fraction at tube outlet $(\hat{z}=1)$

Fig. 8. Bifurcation diagrams versus dimensionless heating power in natural circulation configuration for various spatial discretization (npts $=201$, 401 , 801 and 1601 points), $l=10^{-1} \mathrm{~m}, d=10^{-2} \mathrm{~m}, \rho_{l}=10^{3} \mathrm{~kg} \cdot \mathrm{m}^{-3}, \mu_{l}=10^{-3} \mathrm{~Pa}_{\mathrm{s}} \mathrm{s}^{-1}, \rho_{v}=1 \mathrm{~kg} \cdot \mathrm{m}^{-3} ; \mu_{v}=10^{-5} \mathrm{~Pa}^{-1}, \mathrm{~h}_{l s}=0.419210^{6} \mathrm{~J} \cdot \mathrm{kg}^{-1}, \mathrm{~h}_{v s}=$ $2.675610^{6} \mathrm{~J} . \mathrm{kg}^{-1}, \tilde{G}=544 \mathrm{~kg} \cdot \mathrm{s}^{-1} \cdot \mathrm{m}^{-2}, \tilde{h}=1.04810^{6} \mathrm{~J} . \mathrm{kg}^{-1}, \tilde{q}=310^{8} \mathrm{~W} . \mathrm{m}^{-2}$ and $\varepsilon=10^{-3}$.

Table 1

Spatial convergence analysis of natural circulation configuration at first Hopf bifurcation point $\left(H B_{1}\right)$ for $l=10^{-1} \mathrm{~m}, d=10^{-2} \mathrm{~m}, \rho_{l}=10^{3} \mathrm{~kg} \cdot \mathrm{m}^{-3}$, $\mu_{l}=10^{-3}$ Pa.s ${ }^{-1}, \rho_{v}=1 \mathrm{~kg} \cdot \mathrm{m}^{-3} ; \mu_{v}=10^{-5} \mathrm{~Pa} . \mathrm{s}^{-1}, h_{l s}=0.419210^{6} \mathrm{~J} \cdot \mathrm{kg}^{-1}, h_{v s}=2.675610^{6} \mathrm{~J} \cdot \mathrm{kg}^{-1}, \tilde{G}=544 \mathrm{~kg} \cdot \mathrm{s}^{-1} \cdot \mathrm{m}^{-2}, \tilde{h}=1.04810^{6} \mathrm{~J} \cdot \mathrm{kg}^{-1}$, $\tilde{q}=310^{8} W \cdot m^{-2}$ and $\varepsilon=10^{-3}$.

\begin{tabular}{|c|c|c|c|c|c|c|c|}
\hline Mesh \# & npts & $d z=\hat{l} /(n p t s-1)$ & $\hat{\lambda}_{H B_{1}}$ & $\hat{h}_{m}\left(\hat{l}_{H B_{1}}\right.$ & $\hat{G}_{H B_{1}}$ & $\alpha(\hat{l})_{H B_{1}}$ & $\hat{\omega}_{H B_{1}}$ \\
\hline M1 & 201 & $510^{-3}$ & 0.19371 & 0.392785 & 0.206907 & 0.015701 & \pm 0.61103 \\
\hline M2 & 401 & $2.510^{-3}$ & 0.19353 & 0.392821 & 0.207607 & 0.015779 & \pm 0.61211 \\
\hline M3 & 801 & $1.2510^{-3}$ & 0.19352 & 0.392828 & 0.207763 & 0.015793 & \pm 0.61234 \\
\hline M4 & 1601 & $6.2510^{-4}$ & 0.19351 & 0.392829 & 0.207765 & 0.015796 & \pm 0.61235 \\
\hline \multirow{2}{*}{\multicolumn{3}{|c|}{$\begin{array}{l}\beta=\frac{\ln \left[\left(f_{1}-f_{3}\right) /\left(f_{2}-f_{4}\right)\right]}{\ln \left(d z_{1} / d z_{2}\right)} \\
C_{\beta}=\frac{f_{3}-f_{4}}{d z_{3}^{\beta}-d z_{4}^{\beta}}\end{array}$}} & 3.17 & 2.43 & 2.44 & 2.47 & 2.42 \\
\hline & & & $1.7936210^{4}$ & $-1.3585210^{1}$ & $-2.9273210^{1}$ & $-5.5826510^{1}$ & $-1.3232410^{2}$ \\
\hline \multicolumn{3}{|c|}{$f_{H B_{1}}^{R E}=f_{4}-C_{\beta} d z_{4}^{\beta}$} & 0.19351 & 0.392829 & 0.207765 & 0.015796 & 0.61235 \\
\hline
\end{tabular}

in this region steady-state solutions only exist for decreasing heating power, which nevertheless induces increasing mixture enthalpy in the heated tube. Then, the second turning point occurs at moderate heating power $\left(\hat{\lambda}_{T P_{2}} \approx 0.2028\right)$ and low mass flux across tube $\left(\hat{G}_{T P_{2}} \approx 0.1064\right)$, leading to a mixture enthalpy at tube outlet $\left(\hat{h}_{m}\left(\hat{l}_{T_{P_{2}}} \approx 0.7316\right)\right.$ and high void fraction at tube outlet $\left(\alpha(\hat{l})_{T P_{2}} \approx 0.9945\right)$. Going further beyond this point, the heating power increases again continuously up to single-phase steam $\left(\hat{h}_{v s}=2.553\right)$ and super-heated steam $\left(\hat{h}_{m}>\hat{h}_{v s}\right)$ takes in turn place inside the computational domain.

At first glance all continuation curves corresponding to the various grids considered collapse on a master one. This is true over the three quarters of the whole continuation procedure up to the region where void fraction tends to unity. There, the coarsest grid (npts $=201$ points) is no longer able to capture the problem stiffness, so artificial numerical oscillations appear on its branch of steady-state solutions. On the other hand, all three finer grids both provide smooth oscillation-free converging solutions even in this extremely stiff region, see dimensionless mixture enthalpy at tube outlet, mixture mass flux across tube and void fraction at tube outlet plotted in Figs. 8(a)-8(c), respectively, together with inserts corresponding to close-up view in the vicinity of the two turning points.

\subsubsection{Linear stability changes}

Once branches of steady-state solutions are computed one is interested to find out particular values of control parameter for which stability changes. For this natural circulation configuration, steady-state solutions are linearly unstable in two separate ranges of the control parameter (red lines in Fig. 8). The first one (red dotted-lines) occurs at small void fraction and takes place between two Hopf bifurcation points (red crosses), so that steady-state solutions loose their stability towards oscillating unsteady solutions leading to a so-called dynamical instability. The second region (red solid-lines) appears from moderate to high void fractions and it lies between two turning points (red circles), so that steady-state solutions loose their stability towards steady ones leading to a so-called static instability or Ledinegg instability.

The locations of the two Hopf bifurcation points along with their respective angular frequencies are reported for the four grids considered in Tables 1 and 2, together with Richardson extrapolation parameters (convergence order, constant and extrapolated values). Similarly, locations of the two turning points are reported in Tables 3 and 4, respectively, together with the Richardson extrapolation parameters (convergence order, constant and extrapolated values).

One can observe that convergence orders (designated $\beta$ in Tables 1-4) increase as void fraction gets larger at considered point. This can be explained by looking at the various terms involved in governing equations. Indeed, at very low void fractions, the leading terms in momentum equation are buoyancy and wall friction that do not involve any spatial derivative, inducing a global first-order spatial convergence. At slightly higher void fractions, spatial derivatives of pressure and 


\section{Table 2}

Spatial convergence analysis of natural circulation configuration at second Hopf bifurcation point $\left(H B_{2}\right)$ for $l=10^{-1} \mathrm{~m}, d=10^{-2} \mathrm{~m}, \rho_{l}=10^{3} \mathrm{~kg} \cdot \mathrm{m}^{-3}$ $\mu_{l}=10^{-3}$ Pa.s $\mathrm{s}^{-1}, \rho_{v}=1 \mathrm{~kg} . \mathrm{m}^{-3} ; \mu_{v}=10^{-5} \mathrm{~Pa} . \mathrm{s}^{-1}, h_{l s}=0.419210^{6} \mathrm{~J} . \mathrm{kg}^{-1}, h_{v s}=2.675610^{6} \mathrm{~J} . \mathrm{kg}^{-1}, \tilde{G}=544 \mathrm{~kg} . \mathrm{s}^{-1} . \mathrm{m}^{-2}, \tilde{h}=1.04810^{6} \mathrm{~J} . \mathrm{kg}^{-1}$, $\tilde{q}=310^{8} W \cdot m^{-2}$ and $\varepsilon=10^{-3}$.

\begin{tabular}{|c|c|c|c|c|c|c|c|}
\hline Mesh \# & npts & $d z=\hat{l} /(n p t s-1)$ & $\hat{\lambda}_{H B_{2}}$ & $\hat{h}_{m}(\hat{l})_{H B_{2}}$ & $\hat{G}_{H B_{2}}$ & $\alpha(\hat{l})_{H B_{2}}$ & $\hat{\omega}_{H B_{2}}$ \\
\hline M1 & 201 & $510^{-3}$ & 0.43809 & 0.399262 & 0.461086 & 0.106412 & \pm 0.96050 \\
\hline M2 & 401 & $2.510^{-3}$ & 0.43589 & 0.399286 & 0.461888 & 0.107009 & \pm 0.96061 \\
\hline M3 & 801 & $1.2510^{-3}$ & 0.43563 & 0.399289 & 0.461913 & 0.107097 & \pm 0.96062 \\
\hline M4 & 1601 & $6.2510^{-4}$ & 0.43562 & 0.399291 & 0.462039 & 0.107131 & \pm 0.96063 \\
\hline \multirow{2}{*}{\multicolumn{3}{|c|}{$\begin{array}{l}\beta=\frac{\ln \left[\left(f_{1}-f_{3}\right) /\left(f_{2}-f_{4}\right)\right]}{\ln \left(d z_{1} / d z_{2}\right)} \\
C_{\beta}=\frac{f_{3}-f_{4}}{d z_{3}^{\beta}-d z_{4}^{\beta}}\end{array}$}} & 3.19 & 2.46 & 2.45 & 2.49 & 2.48 \\
\hline & & & $2.0158510^{4}$ & $-3.433310^{1}$ & $-2.042610^{3}$ & $-6.967910^{2}$ & $-1.937410^{2}$ \\
\hline \multicolumn{3}{|c|}{$f_{H B_{2}}^{R E}=f_{4}-C_{\beta} d z_{4}^{\beta}$} & 0.435619 & 0.399291 & 0.462067 & 0.107138 & 0.96083 \\
\hline
\end{tabular}

Table 3

Spatial convergence analysis of natural circulation configuration at first turning point $\left(T P_{1}\right)$ for $l=10^{-1} \mathrm{~m}, d=10^{-2} \mathrm{~m}, \rho_{l}=10^{3} \mathrm{~kg} \cdot \mathrm{m}^{-3}, \mu_{l}=10^{-3}$

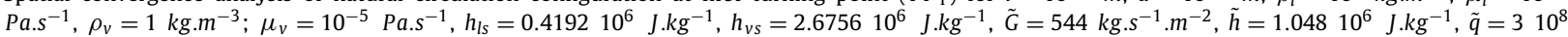
W. $m^{-2}$ and $\varepsilon=10^{-3}$.

\begin{tabular}{|c|c|c|c|c|c|c|}
\hline Mesh \# & npts & $d z=\hat{l} /($ npts -1$)$ & $\hat{\lambda}_{T P_{1}}$ & $\hat{h}_{m}(\hat{l})_{T P_{1}}$ & $\hat{G}_{T P_{1}}$ & $\alpha\left(\hat{l}_{T P_{1}}\right.$ \\
\hline M1 & 201 & $510^{-3}$ & 0.49319440 & 0.40084100 & 0.51717300 & 0.3401070 \\
\hline M2 & 401 & $2.510^{-3}$ & 0.49319595 & 0.40089350 & 0.51715555 & 0.3418950 \\
\hline M3 & 801 & $1.2510^{-3}$ & 0.49319599 & 0.40089495 & 0.51715502 & 0.3419515 \\
\hline M4 & 1601 & $6.2510^{-4}$ & 0.49319600 & 0.40089500 & 0.51715500 & 0.3419520 \\
\hline \multicolumn{3}{|c|}{$\beta=\frac{\ln \left[\left(f_{1}-f_{3}\right) /\left(f_{2}-f_{4}\right)\right]}{\ln \left(d z_{1} / d z_{2}\right)}$} & 5.03 & 5.10 & 5.03 & 5.02 \\
\hline \multicolumn{3}{|c|}{$C_{\beta}=\frac{f_{3}-f_{4}}{d z_{3}^{\beta}-d z^{\beta}}$} & $-4.05836510^{5}$ & $-5.20108110^{7}$ & $4.69598510^{7}$ & $-1.88305110^{8}$ \\
\hline \multicolumn{3}{|c|}{$f_{T P_{1}}^{R E}=f_{4}-C_{\beta} d z_{4}^{\beta}$} & 0.493196 & 0.400895 & 0.517155 & 0.341952 \\
\hline
\end{tabular}

Table 4

Spatial convergence analysis of natural circulation configuration at second turning point $\left(T P_{2}\right)$ for $l=10^{-1} \mathrm{~m}, d=10^{-2} \mathrm{~m}, \rho_{l}=10^{3} \mathrm{~kg} \cdot \mathrm{m}^{-3}, \mu_{l}=10^{-3}$ Pa.s ${ }^{-1}, \rho_{v}=1 \mathrm{~kg} . \mathrm{m}^{-3} ; \mu_{v}=10^{-5} \mathrm{~Pa}_{\mathrm{s}}^{-1}, h_{l s}=0.419210^{6} \mathrm{~J} . \mathrm{kg}^{-1}, h_{v s}=2.675610^{6} \mathrm{~J} . \mathrm{kg}^{-1}, \tilde{G}=544 \mathrm{~kg} . \mathrm{s}^{-1} . \mathrm{m}^{-2}, \tilde{h}=1.04810^{6} \mathrm{~J} . \mathrm{kg}^{-1}, \tilde{q}=310^{8}$ $W . m^{-2}$ and $\varepsilon=10^{-3}$.

\begin{tabular}{llllll}
\hline Mesh \# & npts & $d z=\hat{l} /(n p t s-1)$ & $\hat{\lambda}_{T P_{2}}$ & $\hat{h}_{m}(\hat{l})_{T P_{2}}$ & $\hat{G}_{T P_{2}}$ \\
\hline M1 & 201 & $510^{-3}$ & 0.20275700 & 0.72856600 & 0.1067000 \\
M2 & 401 & $2.510^{-3}$ & 0.20276502 & 0.73162550 & 0.1063555 \\
M3 & 801 & $1.2510^{-3}$ & 0.20276599 & 0.73159700 & 0.1064365 \\
M4 & 1601 & $6.2510^{-4}$ & 0.20276600 & 0.73159688 & 0.1064369 \\
$\beta=\frac{\ln \left[\left(f_{2}-f_{3}\right) /\left(f_{3}-f_{4}\right)\right]}{\ln \left(d z_{1} / d z_{2}\right)}$ & & 5.50 & 5.47 & 5.44 \\
$C_{\beta}=\frac{f_{3}-f_{4}}{d z_{3}^{\beta}-d z_{4}^{\beta}}$ & & $-8.23654410^{5}$ & $2.10278510^{7}$ & $-5.25387210^{7}$ \\
$f_{T P_{2}}^{R E}=f_{4}-C_{\beta} d z_{4}^{\beta}$ & & 0.202766 & 0.99452400 & 0.731597 & 0.106437 \\
\hline
\end{tabular}

acceleration terms enter the game, enabling to increase the global convergence order. Finally at high void fractions, spatial derivatives of acceleration and pressure terms mainly determine the global spatial convergence order, rising up to $\beta \approx 5.5$ at second turning point, where $\alpha$ tends to unity. This value is perfectly consistent with formal orders of spatial derivatives used in this implementation, 6th order for collocated variables at collocated grid points and 5th order otherwise. As spatial convergence has been achieved on a representative case, then one can consider discrete solutions belonging to the range of asymptotic convergence of the method to perform Richardson extrapolation of quantities of interest. They are denoted $f^{R E}$ in Tables 1-4 and are based on the four grids considered for the two Hopf bifurcation points and first turning point whereas they are based on the only three finest ones for the second turning point.

\subsection{Sensitivity to regularization parameter}

As previously mentioned governing equations consist of a regularized void fraction relationship, eq. (13), which provides continuity and derivability properties suitable for our Asymptotic Numerical Method. Therefore, the present sensitivity analysis reports on how the computed results depend on this regularization parameter (designated $\varepsilon$ ). The few other numerical parameters involved with the Asymptotic Numerical Method are the same as those used in our previous works [13,34].

First of all, let us introduce how does the regularization parameter act on a simplified problem. For that purpose, thanks to the Mathematica software [54] one solves for the void fraction from eq. (13) alone, with prescribed dimensionless mass flux, relative vapor-liquid velocity and mixture enthalpy, for three values of the regularization parameter $\left(\varepsilon=10^{-3}, 10^{-4}\right.$ and $\left.10^{-5}\right)$. The resulting void fraction distributions versus dimensionless mixture enthalpy are displayed in 


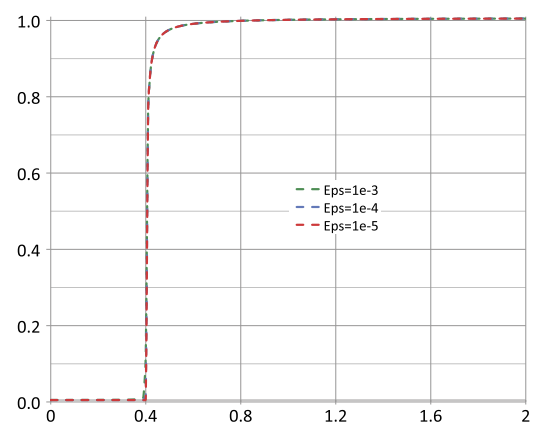

(a) Linear scales.

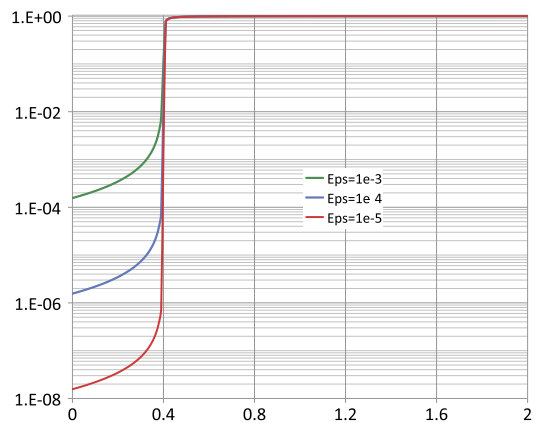

(b) Linear-logarithmic scales.

Fig. 9. Full-range regularized void fraction, eq. (13), versus dimensionless mixture enthalpy for $\hat{h}_{l_{\mathrm{s}}}=0.4, \hat{h}_{l_{\mathrm{s}}}=2.55, \hat{\mathrm{G}}=1, \rho_{l}=10^{3} \mathrm{~kg} \cdot \mathrm{m}^{-3}, \rho_{v}=1 \mathrm{~kg} \cdot \mathrm{m}^{-3}$ and three values of regularization parameter $\varepsilon=10^{-3}, 10^{-4}$ and $10^{-5}$. (a) linear scales; (b) linear-logarithmic scales.

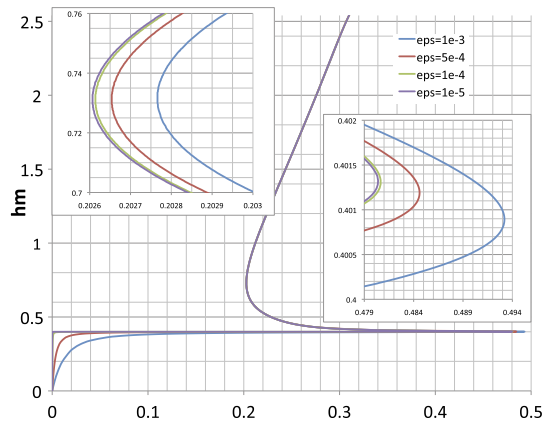

(a) Mixture enthalpy at tube outlet $(\hat{z}=1)$.

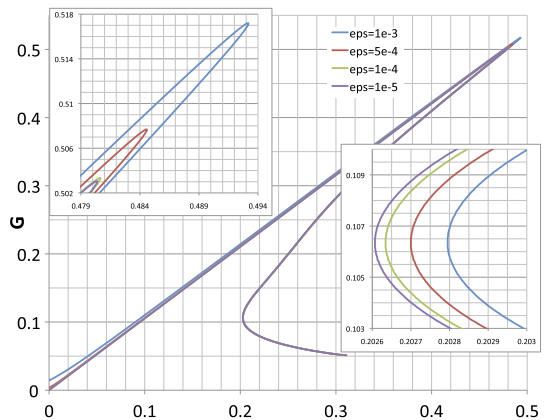

(b) Mixture mass flux across tube.

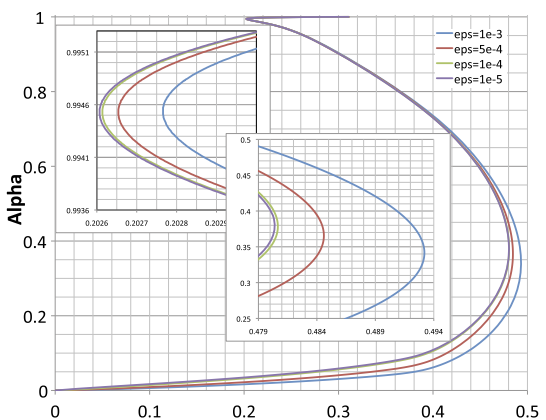

(c) Void fraction at tube outlet $(\hat{z}=1)$.

Fig. 10. Continuation diagrams versus dimensionless heating power in natural circulation configuration for various values of regularization parameter $\left(\varepsilon=10^{-3}, 510^{-4}, 10^{-4}\right.$ and $\left.10^{-5}\right), l=10^{-1} \mathrm{~m}, d=10^{-2} \mathrm{~m}, \rho_{l}=10^{3} \mathrm{~kg} \cdot \mathrm{m}^{-3}, \mu_{l}=10^{-3}$ Pa.s $\mathrm{s}^{-1}, \rho_{v}=1 \mathrm{~kg} \cdot \mathrm{m}^{-3} ; \mu_{v}=10^{-5}$ Pa.s $\mathrm{s}^{-1}, h_{l s}=0.419210^{6}$ $J . \mathrm{kg}^{-1}, h_{v s}=2.675610^{6} \mathrm{~J} . \mathrm{kg}^{-1}, \tilde{G}=544 \mathrm{~kg} \cdot \mathrm{s}^{-1} \cdot \mathrm{m}^{-2}, \tilde{h}=1.04810^{6} \mathrm{~J} \cdot \mathrm{kg}^{-1}, \tilde{q}=310^{8} \mathrm{~W} . \mathrm{m}^{-2}$ and npts $=1601$ points.

Table 5

Values of dimensionless quantities at the two turning points in natural circulation configuration versus regularization parameter, $l=10^{-1} \mathrm{~m}, d=10^{-2}$ $m, \rho_{l}=10^{3} \mathrm{~kg} \cdot \mathrm{m}^{-3}, \mu_{l}=10^{-3} \mathrm{~Pa} . \mathrm{s}^{-1}, \rho_{v}=1 \mathrm{~kg} \cdot \mathrm{m}^{-3} ; \mu_{v}=10^{-5} \mathrm{~Pa} . \mathrm{s}^{-1}, h_{l s}=0.419210^{6} \mathrm{~J} . \mathrm{kg}^{-1}, h_{v s}=2.675610^{6} \mathrm{~J} . \mathrm{kg}^{-1}, \tilde{G}=544 \mathrm{~kg} . \mathrm{s}^{-1} . \mathrm{m}^{-2}$, $\tilde{h}=1.04810^{6} \mathrm{~J} \cdot \mathrm{kg}^{-1}, \tilde{q}=310^{8} \mathrm{~W} \cdot \mathrm{m}^{-2}$ and npts $=1601$ points.

\begin{tabular}{llllllll}
\hline$\varepsilon$ & $\hat{\lambda}_{T P_{1}}$ & $\alpha(\hat{l})_{T P_{1}}$ & $\hat{h}_{m}\left(\hat{l}_{T P_{1}}\right.$ & $\hat{G}_{T P_{1}}$ & $\hat{\lambda}_{T P_{2}}$ & $\alpha\left(\hat{l}_{T P_{2}}\right.$ & $\hat{h}_{m}\left(\hat{l}_{T P_{2}}\right.$ \\
\hline $10^{-3}$ & 0.493196 & 0.341952 & 0.400895 & 0.517155 & 0.202766 & 0.994530 & 0.731231 \\
$510^{-4}$ & 0.484599 & 0.364788 & 0.401185 & 0.507662 & 0.202654 & 0.994528 & 0.731202 \\
$10^{-4}$ & 0.480663 & 0.378471 & 0.401308 & 0.503318 & 0.202614 & 0.994527 & 0.731199 \\
$10^{-5}$ & 0.480394 & 0.379052 & 0.401313 & 0.503027 & 0.202607 & 0.994526 & 0.731196406340 \\
\hline
\end{tabular}

Figs. 9(a) and 9(b), on linear and logarithmic scales, respectively. It clearly appears that the influence of regularization parameter is only restricted to the sub-cooled region $\left(\hat{h}_{m}<\hat{h}_{l_{s}}=0.4\right)$, where the theoretical value of void fraction is obviously zero whereas the regularized one does not. This latter tends to zero as mixture enthalpy decreases from liquid saturation enthalpy and its magnitude behaves as $\varepsilon^{2}$, so reducing the regularization parameter by one order diminishes the regularized void fraction by two orders, as depicted in Fig. 9(b).

Then, to analyze the global influence of regularization parameter on our model let us move to the numerical solution of the fully coupled set of governing equations, considering the same natural circulation configuration as in previous subsection. Branches of steady-state solutions are plotted in Fig. 10 for four values of the regularization parameter $\left(\varepsilon=10^{-3}\right.$, $510^{-4}, 10^{-4}$ and $10^{-5}$ ) and a refined spatial resolution (npts $=1601$ points). One can notice as in the simplified problem that the influence of the regularization parameter appears mainly at the lowest heating powers leading to sub-cooled conditions and small to moderate void fractions. Then, at higher void fractions, a very fast convergence is achieved versus regularization parameter, as clearly displayed by comparing branches of steady-state solutions computed with $\varepsilon=10^{-4}$ to those with $10^{-5}$, since their relative deviation is less than $\varepsilon$ itself.

Quantitative comparisons of dimensionless values at first and second turning points have been reported in Table 5, moreover, they are plotted versus regularization parameter in Figs. 11(a) and 11(b), respectively. It is noteworthy that in this dimensionless framework values of regularization parameter lower than $\varepsilon=510^{-4}$ produces very accurate results. However, it should be mentioned that the lower $\varepsilon$ the higher the computational costs of the continuation procedure as the step length reduces with the nonlinear stiffness of the problem. The computational cost (CPU time) is twice from $\varepsilon=10^{-3}$ 


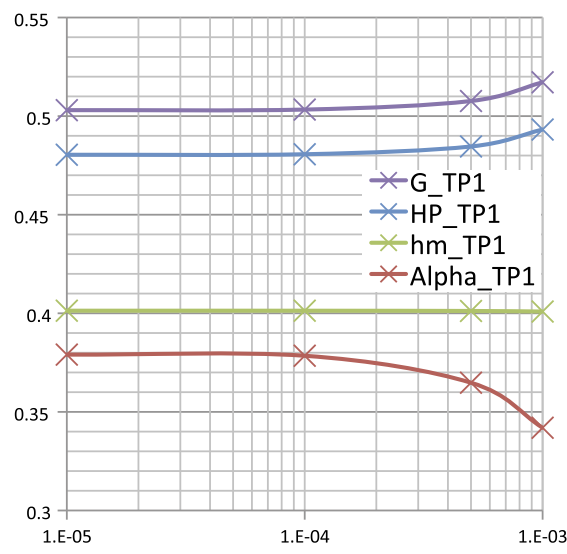

(a) Values at first turning point.

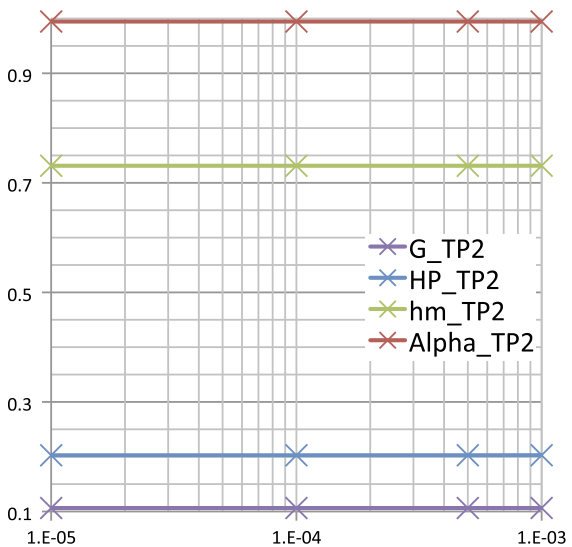

(b) Values at second turning point.

Fig. 11. Dimensionless quantities at the two turning points in natural circulation configuration versus regularization parameter $\left(\varepsilon=10^{-3}, 510^{-4}, 10^{-4}\right.$ and $\left.10^{-5}\right), l=10^{-1} \mathrm{~m}, d=10^{-2} \mathrm{~m}, \rho_{l}=10^{3} \mathrm{~kg} \cdot \mathrm{m}^{-3}, \mu_{l}=10^{-3} \mathrm{~Pa} \cdot \mathrm{s}^{-1}, \rho_{v}=1 \mathrm{~kg} \cdot \mathrm{m}^{-3} ; \mu_{v}=10^{-5} \mathrm{~Pa}^{-1}, \mathrm{~h}_{l s}=0.419210^{6} \mathrm{~J} . \mathrm{kg}^{-1}, h_{v s}=2.675610^{6}$ $J . \mathrm{kg}^{-1}, \tilde{G}=544 \mathrm{~kg} \cdot \mathrm{s}^{-1} \cdot \mathrm{m}^{-2}, \tilde{h}=1.04810^{6} \mathrm{~J} \cdot \mathrm{kg}^{-1}, \tilde{q}=310^{8} \mathrm{~W} \cdot \mathrm{m}^{-2}$ and npts $=1601$ points.

to $510^{-4}$ and the same overhead comes again from $510^{-4}$ to $10^{-4}$, but no significant further cost increase is observed from that latter value to the smaller one considered, $\varepsilon=10^{-5}$.

\section{Conclusion}

This paper presents a methodological approach that aims to bring altogether advanced nonlinear algorithms and linear stability ones into a single numerical model devoted to study boiling channel flows. The one-dimensional phase-change model considered in this work is based on three drift-flux based conservation equations for the two-phase mixture, supplemented with a specifically derived void fraction equation, along with simple and general-purpose constitutive equations as closures. These governing equations have been derived to suit continuity and derivability requirements of the present high-order model. However, the authors are aware of the various limitations of the present model, but the intended goal is first to show the potential added-value of the present approach on academic configurations.

To take full advantage of the present semi-analytical continuation algorithm, several implementation precautions have be taken to fulfill the desired continuity and derivability properties. First-order spatial derivatives that appear in conservation equations are discretized with high-order finite difference schemes: 6th order for collocated fields at collocated grid point and 5th order elsewhere. Then, spatial convergence analyses have been performed to assess the numerical model implementation. The Richardson extrapolation technique confirmed that in representative natural circulation boiling flows, the overall effective convergence order actually tends towards the formal one, as in best cases it reaches roughly up to $\beta \approx 5.5$.

In the framework of natural circulation boiling channels the present work's strong point is definitely the computation of branches of steady-state solutions (response curves) versus tube heating power. Unlike previous semi-analytical models [1, $30,31,47,55]$, the present approach enables to accurately and efficiently solve the highly nonlinear partial differential equations associated with the fully implicit governing equations along response curves. It follows the Dynamical System Theory in the way one first computes branches of fixed points and then use some of these fixed point solutions as base-state to perform linear stability analyses.

Some added-value of the present approach compared to classical ones is: (i) the developed continuation algorithm enables to compute stable as well as unstable steady-states whereas time integration approaches only compute stable ones; (ii) solving for the fully nonlinear governing equations PDEs provides some insight into the spatial field evolution along the channel together with their spatial instability modes; (iii) the developed approach provides a good computational efficiency that results from judicious combination of semi-analytical Asymptotic Numerical Method and Automatic Differentiation. Besides, their implementation in the Matlab software [33] using operator overloading results in a powerful, efficient and user-friendly scientific tool [5]. Moreover, configurations of mixed forced and natural circulation can also be computed by using the driving pressure difference at tube ends as continuation parameter.

Finally, the present model suffers from several weaknesses that could be improved in forthcoming work. The main one is definitely the singularity of the generalized eigenvalue problem that arises in linear stability analyses. Indeed, the inherent problem singularity unavoidably requires out-of-the shelves numerical algorithm to extract the only physically relevant eigenvalues and discard infinite ones. On the other hand, one can extend the present model capabilities toward more physically sounding ones by accounting for compressibility of density vapor phase and pressure dependance of phase-change properties along saturation curves. Also, problem specific constitutive relationships proposed in the literature $[24,25]$ could also be implemented to address relevant targeted configurations, provided one performs some adaptations to match semianalytical requirements of the Asymptotic Numerical Method. Another promising direction of forthcoming work could be to take advantage of the present model that directly integrates the fully nonlinear governing PDEs to provide data to surrogate 
or reduced order models suitable to perform efficiently numerous parametric studies. Finally, in this framework of boiling channels it seems also very promising to perform the continuation of periodic solutions in their fully nonlinear regime and to study their stability.

\section{Declaration of competing interest}

The authors declare that they have no known competing financial interests or personal relationships that could have appeared to influence the work reported in this paper.

\section{Acknowledgement}

This work benefited from a research agreement between Aix-Marseille University and the CEA Cadarache-SFR R\&D project. Project leaders from both sides who promoted this collaborating framework are acknowledged. Moreover, the authors are thank-full to Chenxi Hou, a MSc student from École Polytechnique (Palaiseau, France), who contributed to this work during his research internship at the CEA.

\section{Appendix A. Conservation equations for 1D liquid-vapor flows}

\section{A.1. Mass conservation equations}

For each fluid phase (liquid, subscript $l$ or vapor, subscript $v$ ), the mass conservation equation reads as follows:

$$
\begin{aligned}
\frac{\partial\left(\alpha_{l} \rho_{l}\right)}{\partial t}+\frac{\partial\left(\alpha_{l} \rho_{l} u_{l}\right)}{\partial z} & =-\dot{M}_{l v} \\
\frac{\partial\left(\alpha_{v} \rho_{v}\right)}{\partial t}+\frac{\partial\left(\alpha_{v} \rho_{v} u_{v}\right)}{\partial z} & =\dot{M}_{l v}
\end{aligned}
$$

where $t$ is time, $z$ the axial abscissa, $\alpha_{i}$ the cross-sectional average fraction of fluid phase $i$ ( $\left.i=l, v\right), \rho_{i}$ its density, $u_{i}$ is its 1 D cross-sectional average velocity and $\dot{M}_{l v}$ is the mass flow rate across the liquid-vapor interface associated with phase-change. As defined, the cross-sectional average liquid and vapor fractions make a partition of unity, so that they sum up to unity:

$$
\alpha_{l}+\alpha_{v}=1
$$

Summing up equations (A.1) and (A.2) and substituting the partition of unity relationship (A.3), while designating $\alpha$ as the cross-sectional average void (vapor) fraction, one obtains the mass conservation equation of the liquid-vapor mixture:

$$
\frac{\partial\left[(1-\alpha) \rho_{l}+\alpha \rho_{v}\right]}{\partial t}+\frac{\partial\left[(1-\alpha) \rho_{l} u_{l}+\alpha \rho_{v} u_{v}\right]}{\partial z}=0
$$

\section{A.2. Momentum conservation equations}

For each of the liquid or vapor phases, the momentum conservation equation in projection along the ascending vertical direction reads as follows:

$$
\begin{gathered}
\frac{\partial\left(\alpha_{l} \rho_{l} u_{l}\right)}{\partial t}+\frac{\partial\left(\alpha_{l} \rho_{l} u_{l}^{2}\right)}{\partial z}=-\alpha_{l} \frac{\partial p_{l}}{\partial z}-\beta_{l} \tau_{l} \frac{P}{A}-\alpha_{l} \rho_{l} g-F_{i_{l v}} \\
\frac{\partial\left(\alpha_{v} \rho_{v} u_{v}\right)}{\partial t}+\frac{\partial\left(\alpha_{v} \rho_{v} u_{v}^{2}\right)}{\partial z}=-\alpha_{v} \frac{\partial p_{v}}{\partial z}-\beta_{v} \tau_{v} \frac{P}{A}-\alpha_{v} \rho_{v} g+F_{i_{l v}}
\end{gathered}
$$

where $p_{i}$ designates pressure in the $i$ phase, $\tau_{i}$ is the viscous stress of the $i$ phase at tube wall, $\beta_{i}$ is the perimeter (P) fraction wetted by the $i$ phase and $A$ is the constant cross section of the tube. Moreover, $F_{i_{l v}}$ is the interaction force between phases acting at the liquid-vapor interface.

Let us now consider some simplifying physical assumptions that enables to reduce the number of unknowns. First of all, in this 1D model one has no access to any geometrical information about the liquid-vapor interface. Therefore, the simplest realistic choice is to assume a pressure equilibrium across the interface, leading to: $p_{l}=p_{v}=p_{m}$. Furthermore, the perimeter fractions wetted by liquid and vapor phases make a partition of unity, so that they also sum up to unity:

$$
\beta_{l}+\beta_{v}=1
$$

Summing up equations (A.5) and (A.6) and substituting the partition of unity relationship (A.7) while designating $\beta$ as the perimeter fraction wetted by the vapor phase, one obtains the momentum conservation equation of the liquid-vapor mixture: 


$$
\begin{array}{r}
\frac{\partial\left[(1-\alpha) \rho_{l} u_{l}+\alpha \rho_{v} u_{v}\right]}{\partial t}+\frac{\partial[}{\partial z}= \\
-\frac{\partial p_{m}}{\partial z}-\left[(1-\beta) \tau_{l}+\beta \tau_{v}\right] \frac{P}{A}-\left[(1-\alpha) \rho_{l}+\alpha \rho_{v}\right] g
\end{array}
$$

The second term on the left hand side of eq. (A.8) can be judiciously written as a function of the mixture mass flux $(G)$ and the relative velocity between liquid and vapor phases (designated $U_{r_{v l}}$ ). These two later quantities are related to phase velocities as follows:

$$
\left\{\begin{aligned}
(1-\alpha) \rho_{l} u_{l}+\alpha \rho_{v} u_{v} & =G \\
-u_{l}+u_{v} & =U_{r_{v l}}
\end{aligned}\right.
$$

Therefore, solving this set of two equations gives the two phase velocity relationships:

$$
\begin{aligned}
& u_{l}=\frac{G-\alpha \rho_{v} U_{r_{v l}}}{\rho_{m}} \\
& u_{v}=\frac{G+(1-\alpha) \rho_{l} U_{r_{v l}}}{\rho_{m}}
\end{aligned}
$$

where $\rho_{m}$ is the mixture density defined in eq. (1).

\section{A.3. Energy conservation equations}

According to the first law of Thermodynamics, for each of the liquid or vapor phases, the total energy conservation equation reads as follows:

$$
\begin{gathered}
\frac{\partial\left(\alpha_{l} \rho_{l} e_{l}\right)}{\partial t}+\frac{\partial\left(\alpha_{l} \rho_{l} u_{l} e_{l}\right)}{\partial z}=\beta_{l} q_{w} \frac{P}{A}-\alpha_{l} \frac{\partial\left(p_{l} u_{l}\right)}{\partial z}-\beta_{l} \tau_{l} \frac{P}{A} u_{l}-\alpha_{l} \rho_{l} g u_{l}-P_{i_{l v}}^{t} \\
\frac{\partial\left(\alpha_{v} \rho_{v} e_{v}\right)}{\partial t}+\frac{\partial\left(\alpha_{v} \rho_{v} u_{v} e_{v}\right)}{\partial z}=\beta_{v} q_{w} \frac{P}{A}-\alpha_{v} \frac{\partial\left(p_{v} u_{v}\right)}{\partial z}-\beta_{v} \tau_{v} \frac{P}{A} u_{v}-\alpha_{v} \rho_{v} g u_{v}+P_{i_{l v}}^{t}
\end{gathered}
$$

where $P_{i_{l v}}^{t}$ is the total power exchanged across the liquid-vapor interface. As the total energy is the sum of internal and kinetic energies $\left(e=e^{i}+\frac{u^{2}}{2}\right)$, let us now derive conservation equations for the kinetic energy of each phase.

$$
\begin{gathered}
\frac{\partial\left(\alpha_{l} \rho_{l} \frac{u_{l}^{2}}{2}\right)}{\partial t}+\frac{\partial\left(\alpha_{l} \rho_{l} u_{l}^{2}\right)}{\partial z} u_{l}=-\alpha_{l} \frac{\partial p_{l}}{\partial z} u_{l}-\beta_{l} \tau_{l} \frac{P}{A} u_{l}-\alpha_{l} \rho_{l} g u_{l}-F_{i_{l v}} u_{l} \\
\frac{\partial\left(\alpha_{v} \rho_{v} \frac{u_{v}^{2}}{2}\right)}{\partial t}+\frac{\partial\left(\alpha_{v} \rho_{v} u_{v}^{2}\right)}{\partial z} u_{v}=-\alpha_{v} \frac{\partial p_{v}}{\partial z} u_{v}-\beta_{v} \tau_{v} \frac{P}{A} u_{v}-\alpha_{v} \rho_{v} g u_{v}+F_{i_{l}} u_{v}
\end{gathered}
$$

Therefore, the conservation equations of internal energy can be obtained by subtracting conservation equations of kinetic energy (eq. (A.14)-(A.15)) from those of total energy (eq. (A.12)-(A.13)) as follows:

$$
\begin{gathered}
\frac{\partial\left(\alpha_{l} \rho_{l} e_{l}^{i}\right)}{\partial t}+\frac{\partial\left(\alpha_{l} \rho_{l} u_{l} e_{l}^{i}\right)}{\partial z}=\beta_{l} q_{w} \frac{P}{A}-\alpha_{l} p_{l} \frac{\partial u_{l}}{\partial z}-P_{i_{l v}}^{t}+F_{i_{l v}} u_{l} \\
\frac{\partial\left(\alpha_{v} \rho_{v} e_{v}^{i}\right)}{\partial t}+\frac{\partial\left(\alpha_{v} \rho_{v} u_{v} e_{v}^{i}\right)}{\partial z}=\beta_{v} q_{w} \frac{P}{A}-\alpha_{v} p_{v} \frac{\partial u_{v}}{\partial z}+P_{i_{l v}}^{t}-F_{i_{l v}} u_{v}
\end{gathered}
$$

Introducing the enthalpy expression $\left(h=e^{i}+\frac{p}{\rho}\right)$ into these relationships, the conservation equations for the enthalpy reads for each phase:

$$
\begin{array}{r}
\frac{\partial\left(\alpha_{l} \rho_{l} h_{l}-\alpha_{l} p_{l}\right)}{\partial t}+\frac{\partial\left(\alpha_{l} \rho_{l} u_{l} h_{l}\right)}{\partial z}-u_{l} \frac{\partial\left(\alpha_{l} p_{l}\right)}{\partial z}=\beta_{l} q_{w} \frac{P}{A}-P_{i_{l v}}^{t}+F_{i_{l v}} u_{l} \\
\frac{\partial\left(\alpha_{v} \rho_{v} h_{v}-\alpha_{v} p_{v}\right)}{\partial t}+\frac{\partial\left(\alpha_{v} \rho_{v} u_{v} h_{v}\right)}{\partial z}-u_{v} \frac{\partial\left(\alpha_{v} p_{v}\right)}{\partial z}=\beta_{v} q_{w} \frac{P}{A}+P_{i_{l v}}^{t}-F_{i_{l v}} u_{v}
\end{array}
$$

Summing up equations (A.18) and (A.18) and substituting the partition of unity relationships (A.3) and (A.7), one obtains the enthalpy conservation equation for the liquid-vapor mixture:

$$
\begin{array}{r}
\frac{\partial\left[(1-\alpha) \rho_{l} h_{l}+\alpha \rho_{v} h_{v}-p_{m}\right]}{\partial t}+\frac{\partial\left[(1-\alpha) \rho_{l} u_{l} h_{l}+\alpha \rho_{v} u_{v} h_{v}\right]}{\partial z} \\
-\left[(1-\alpha) u_{l}+\alpha u_{v}\right] \frac{\partial p_{m}}{\partial z}=q_{w} \frac{P}{A}-F_{i_{l v}} U_{r_{v l}}
\end{array}
$$




\section{Appendix B. Closure equations for 1D liquid-vapor flows}

\section{B.1. Kinematic constitutive relationship}

Assuming the constitutive relationship of eq. (14) [59] to hold for given drift velocity $v_{D}$ and distribution parameter $C_{0}$, one can get the expressions of liquid-vapor relative velocity by inserting the expressions of liquid and vapor velocities (eq. (A.10) and eq. (A.11), respectively) into eq. (14). It reads as follows:

$$
U_{r_{v l}}=\frac{G\left(C_{0}-1\right)+\rho_{m} v_{D}}{(1-\alpha)\left[\rho_{l}-\alpha C_{0}\left(\rho_{l}-\rho_{v}\right)\right]}
$$

Plugging back this expression into the original ones (eq. (A.10) and eq. (A.11)), one ends up with liquid and vapor velocity written in function of drift velocity $v_{D}$ and distribution parameter $C_{0}$, as follows:

$$
\begin{aligned}
u_{l} & =\frac{G\left(1-\alpha C_{0}\right)-\alpha \rho_{v} v_{D}}{(1-\alpha)\left[\rho_{l}-\alpha C_{0}\left(\rho_{l}-\rho_{v}\right)\right]} \\
u_{v} & =\frac{G C_{0}+\rho_{l} v_{D}}{\left(1-\alpha C_{0}\right) \rho_{l}+\alpha C_{0} \rho_{v}}
\end{aligned}
$$

To derive a full-range model (that holds for $\alpha \in[0,1]$ ) from expressions of eq. ((B.1)-(B.3)), one has to satisfy some physical and algebraic conditions. Let us begin with the two single-phase limits: mainly liquid $(\alpha \rightarrow 0)$ and mainly vapor $(\alpha \rightarrow 1)$, respectively. Indeed, at these two limit-cases one can show from basic force balance that the liquid-vapor relative velocity should fade away. Let us first consider the former case for which one assumes a bubbly flow, such that vapor bubbles are sufficiently dispersed in the liquid phase that their collective effects are negligible $(0<\alpha \ll 1)$. Then, let us assume there are $n$ small enough bubbles to be spherical. In the opposite mist-flow limit, just below the saturated vapor limit $(\alpha \rightarrow 1)$, a similar reasoning can be conducted considering sufficiently small liquid droplets to be spherical and well dispersed into the vapor phase. The steady-state relative velocity between vapor bubbles (former case) or liquid droplets (latter case) and surrounding fluid (liquid and vapor, respectively) results from the balance between drag force and buoyancy. Thus, in these two limit-cases, the relative liquid-vapor velocity reads:

$$
\begin{aligned}
& U_{r_{v l}}(\alpha \rightarrow 0)=\lim _{\alpha \rightarrow 0}\left[\frac{4 d\left(\rho_{l}-\rho_{v}\right) g}{3 \rho_{l} C_{D}}(1-\alpha)\left(\frac{\alpha}{n}\right)^{\frac{1}{2}}\right]^{\frac{1}{2}}=0 \\
& U_{r_{v l}}(\alpha \rightarrow 1)=\lim _{\alpha \rightarrow 1}\left[\frac{4 d\left(\rho_{l}-\rho_{v}\right) g}{3 \rho_{v} C_{D}} \alpha\left(\frac{1-\alpha}{n}\right)^{\frac{1}{2}}\right]^{\frac{1}{2}}=0
\end{aligned}
$$

where $d$ is the tube diameter, $g$ is gravity and $C_{D}$ is the bubble or droplet drag coefficient. Inserting in turn eq. (B.4) and eq. (B.5) into eq. (B.1) produces the two limit conditions:

$$
\begin{aligned}
& v_{D}(\alpha=0)=v_{D}(\alpha=1)=0 \\
& C_{0}(\alpha=0)=C_{0}(\alpha=1)=1
\end{aligned}
$$

The second condition that has to be satisfied for getting bounded fluid flow velocities from eq. ((B.1)-(B.3)) is that the distribution parameter $C_{0}$ should be bounded as follows:

$$
1 \leq C_{0}(\alpha) \leq \frac{\rho_{l}}{\rho_{l}-\rho_{v}}
$$

Once we have ruled out the basic features a full-range constitutive equation should satisfy, we have then looked for a drift velocity expression that accommodates the two end-limit conditions of eq. (B.6). The analytical expression that fits all these requirements reads:

$$
v_{D}=v_{D_{0}}\left[\tanh \left(a_{v} \alpha\right)-\tanh \left(b_{v}(\alpha-1)\right)-1\right]\left(1-\alpha^{c_{v}}\right)
$$

where $v_{D_{0}}=\frac{9}{16} \sqrt{\frac{g d\left(\rho_{l}-\rho_{v}\right)}{\rho_{l}}}$ is the drift velocity drawn from [50] when $\alpha$ tends to zero and $C_{0}=1$. The three constants that it contains have been obtained by substituting eq. (B.9) into eq. (B.1) and satisfying the two limit conditions of eq. (B.6) and eq. (B.7). Their values are: $a_{v}=35, b_{v}=18$ and $c_{v}=45$, respectively. 


\section{Appendix C. Finite difference discretization schemes}

For such one-dimensional problem discretization coefficients of first-order derivatives involved in the governing equations have been obtained according to the classical finite difference method. Stencil coefficients at current grid point $i$ have been derived at prescribed order, denoted $m$, in four steps. In a first step, one derives at $m$ neighbor grid points Taylor series expansions of the unknown up to its $m$ th order derivatives. Second step, every equation is multiplied by a coefficient associated with the corresponding $m+1$ grid points. Third step, summing up coefficients at each order of the Taylor series expansion, equating to one the sum associated to first order and to zero all other ones, it results a set of $m+1$ linear equations for the stencil coefficients. Fourth step, solving this set of linear equations with a symbolic linear algebra software, e.g., Xcas a free online one [41], one obtains the literal expression of sought stencil coefficients. Applying this procedure up to 6th order for collocated variables at collocated grid points and 5th order elsewhere, in a centered way apart from domain ends, forward and backward in vicinity of left and right boundaries, respectively, led us to the following stencil coefficients, cf. Fig. 7 to refer to variable notation on the considered staggered grids.

\section{C.1. Discrete first-order derivative at collocated grid points of collocated variables $\left(G, h_{m}, \alpha\right)$}

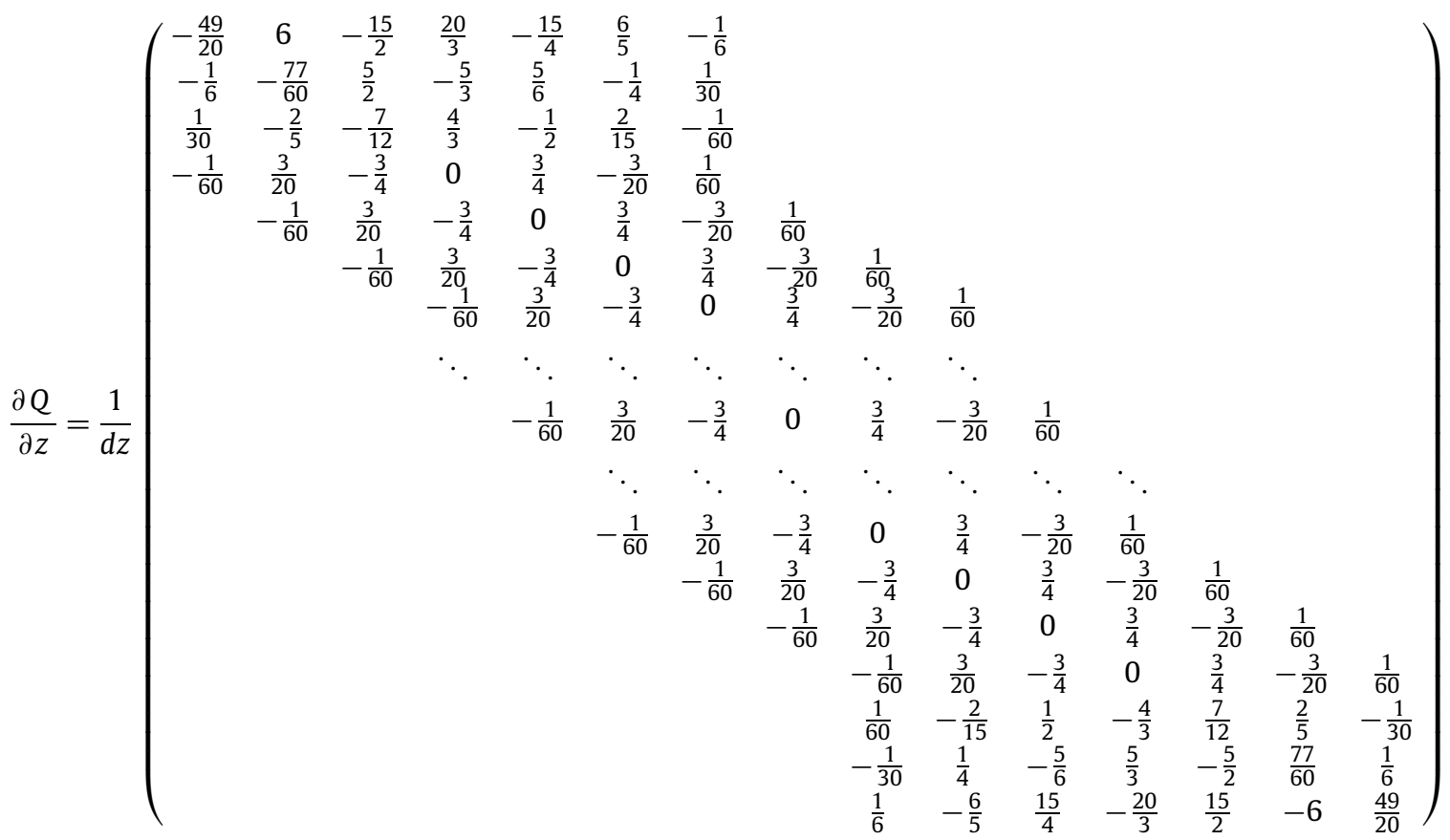

$$
\times\left\{\begin{array}{c}
Q_{1} \\
Q_{2} \\
Q_{3} \\
Q_{4} \\
Q_{5} \\
Q_{6} \\
Q_{7} \\
\vdots \\
Q_{i} \\
\vdots \\
Q_{n-6} \\
Q_{n-5} \\
Q_{n-4} \\
Q_{n-3} \\
Q_{n-2} \\
Q_{n-1} \\
Q_{n}
\end{array}\right\}
$$

where $d z=\hat{l} /(n-1)$ is the uniform space increment separating $n$ evenly distributed points along the tube. 
C.2. Discrete first-order derivative at collocated grid points of mixture pressure (momentum equation)

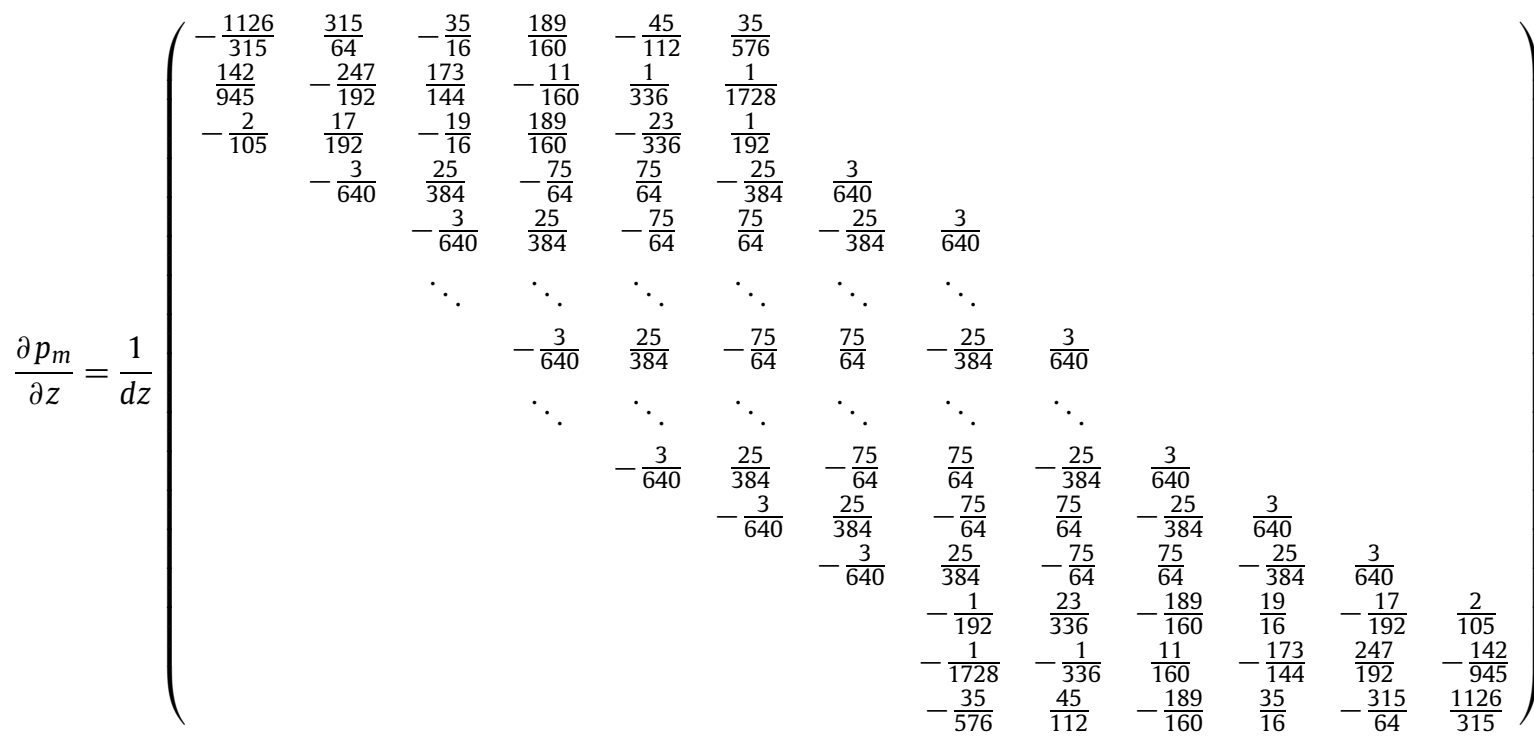

$$
\begin{aligned}
& \times\left\{\begin{array}{c}
p_{0} \\
p_{1} \\
p_{2} \\
p_{3} \\
p_{4} \\
p_{5} \\
\vdots \\
p_{i} \\
\vdots \\
p_{n-5} \\
p_{n-4} \\
p_{n-3} \\
p_{n-2} \\
p_{n-1} \\
p_{L}
\end{array}\right\}
\end{aligned}
$$

C.3. Discrete first-order derivative at staggered grid points of mixture mass flux (mass conservation equation)

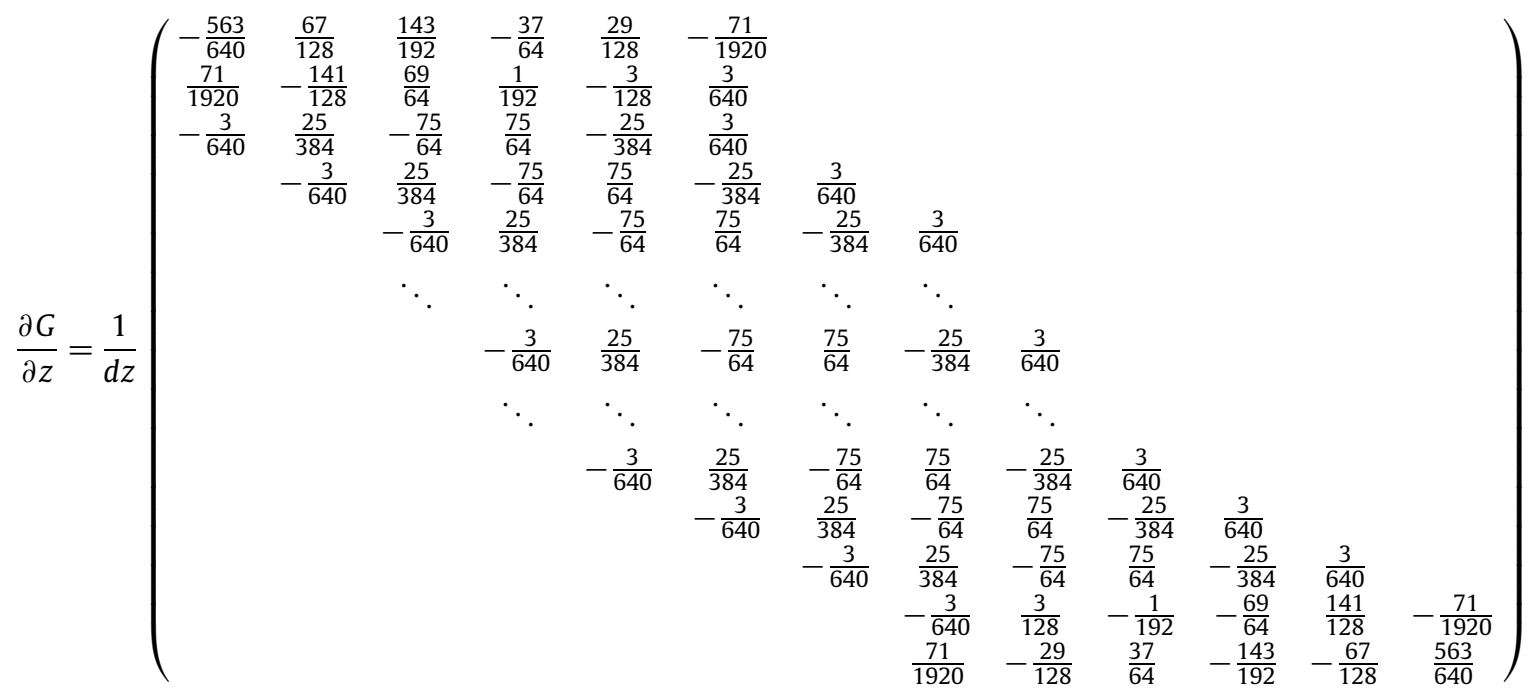




$$
\times\left\{\begin{array}{c}
G_{1} \\
G_{2} \\
G_{3} \\
G_{4} \\
G_{5} \\
G_{6} \\
\vdots \\
G_{i} \\
\vdots \\
G_{n-5} \\
G_{n-4} \\
G_{n-3} \\
G_{n-2} \\
G_{n-1} \\
G_{n}
\end{array}\right\}
$$

\section{References}

[1] J. Achard, D.A. Drew, R.T. Lahey, The analysis of nonlinear density-wave oscillations in boiling channels, J. Fluid Mech. 155 (1985) $213-232$.

[2] E.L. Allgower, K. Georg, Continuation an path following, Acta Numer. 2 (1993) 1-64.

[3] N. Alpy, P. Marsault, M. Anderhuber, A. Gerschenfeld, P. Sciora, D. Kadri, J. Perez, R. Lavastre, Phenomenological investigation of sodium boiling in a SFR core during a postulated ULOF transient with CATHARE 2 system code: a stabilized boiling case, J. Nucl. Sci. Technol. 53 (2016) $692-697$.

[4] J.A. Bouré, A.E. Bergles, L.S. Tong, Review of two-phase flow instability, Nucl. Eng. Des. 25 (1973) $162-192$.

[5] I. Charpentier, B. Cochelin, K. Lampoh, Diamanlab - an interactive Taylor-based continuation tool in Matlab, https://hal.archives-ouvertes.fr/hal00853599.

[6] I. Charpentier, B. Cochelin, Towards a full higher order AD-based continuation and bifurcation framework friction, Optim. Methods Softw. (2018) 1-18, https://doi.org/10.1080/10556788.2018.1428604.

[7] S.W. Churchill, Friction factor equation spans all fluid-flow regimes, Chem. Eng. 84 (1977) 91-92.

[8] K.A. Cliffe, T.J. Garratt, A. Spence, Eigenvalues of the discretized Navier-Stokes equation with application to the detection of Hopf bifurcations, Adv. Comput. Math. 1 (1993) 337-356

[9] K.A. Cliffe, T.J. Garratt, A. Spence, Eigenvalues of block matrices arising from problems in fluid mechanics, SIAM J. Matrix Anal. Appl. 15 (1994) $1310-1318$.

[10] K.A. Cliffe, A. Spence, S.J. Tavener, The numerical analysis of bifurcation problems with application to fluid mechanics, Acta Numer. 9 (2000) 39-131, 2008.

[11] B. Cochelin, A path following technique via an asymptotic numerical method, Comput. Struct. 53 (1994) 1181-1192.

[12] B. Cochelin, N. Damil, M. Potier-Ferry, Méthode Asymptotique Numérique, Hermès - Lavoisier, 2007 (in french)

[13] B. Cochelin, M. Medale, Power series analysis as a major breakthrough to improve the efficiency of asymptotic numerical method in the vicinity of bifurcations, J. Comput. Phys. 236 (2013) 594-607.

[14] H.A. Dijkstra, F.W. Wubs, A.K. Cliffe, E. Doedel, I.F. Dragomirescu, B. Eckhardt, A.Y. Gelfgat, A.L. Hazel, V. Lucarini, A.G. Salinger, E.T. Phipps, J. Sanchez Umbria, H. Schuttelaars, L.S. Tuckerman, U. Thiele, Numerical bifurcation methods and their application to fluid dynamics: analysis beyond simulation, Commun. Comput. Phys. 15 (2014) 1-45.

[15] E. Doedel, H.B. Keller, J.P. Kernevez, Numerical analysis and control of bifurcation problems (i) bifurcation in finite dimensions, Int. J. Bifurc. Chaos 3 (1991) 493-520.

[16] E. Doedel, Nonlinear numerics, J. Franklin Inst. 334B (1997) 1049-1073.

[17] E. Doedel, Lecture notes on numerical analysis of nonlinear equations, in: B. Krauskopf, H. Osinga, J. Galan-Vioque (Eds.), Numerical Continuation Methods for Dynamical Systems, Springer, 2007, pp. 1-49.

[18] A. Dokhane, D. Hennig, R. Chawla, Rizwan-Uddin, Semi-analytical bifurcation analysis of two-phase flow in a heated channel, Int. J. Bifurc. Chaos 15 ( 8 ) (2005) 2395-2409.

[19] P.G. Draizin, W.H. Reid, Hydrodynamic Stability, Cambridge University Press, 1981

[20] D.R. Fokkema, G.L.G. Sleijpen, H.A. van der Vorst, Jacobi-Davidson style QR and QZ algorithms for the reduction of matrix pencils, SIAM J. Sci. Comput. 20 (1) (1998) 94-125

[21] K. Fukuda, T. Kobori, Classification of two-phase flow instability by density wave oscillation model, J. Nucl. Sci. Technol. 16 (2) (1979) 95-108.

[22] A. Griewank, Evaluating Derivatives: Principles and Techniques of Algorithmic Differentiation, Frontiers in Applied Mathematics, SIAM, Philadelphia, 2000.

[23] F.H. Harlow, J.E. Welch, Numerical calculation of time-dependent viscous incompressible flow of fluid with a free surface, Phys. Fluids 8 (1965 $2182-2189$.

[24] T. Hibiki, M. Ishii, One-dimensional drift-flux model and constitutive equations for relative motion between phases in various two-phase flow regimes, Int. J. Heat Mass Transf. 46 (25) (2003) 4935-4948.

[25] T. Hibiki, M. Ishii, Erratum to: "One-dimensional drift-flux model and constitutive equations for relative motion between phases in various two-phase flow regimes”, Int. J. Heat Mass Transf. 46 (2003) 4935-4948, Int. J. Heat Mass Transf. 48 (6) (2005) 1222-1223.

[26] Passive Safety Systems and Natural Circulation in Water Cooled Nuclear Power Plants, TECDOC Series, vol. 1624, International Atomic Energy Agency, Vienna, 2009.

[27] G. Iooss, D.D. Joseph, Elementary Stability and Bifurcation Theory, Springer, New York, 1981.

[28] M. Ishii, One-Dimensional Drift-Flux Model and Constitutive Equations for Relative Motion Between Phases in Various Two-Phase Flow Regimes, Technical Report ANL-77-47, Argonne National Lab. Ill. (USA), 1977, https://doi.org/10.2172/6871478.

[29] H.B. Keller, Numerical Solution of Bifurcation and Nonlinear Eigenvalue Problems, Academic Press, New York, 1977. 
[30] R.T. Lahey, G. Yadigaroglu, A Lagrangian analysis of two-phase hydrodynamic and nuclear-coupled density-wave oscillations, in: Proceedings of the Fifth Int. Heat Transfer Conf., September 3-7, 1974, Tokyo, IV, 1974, B5.9.G.

[31] C. Lange, D. Hennig, A. Hurtado, An advanced reduced order model for BWR stability analysis, Prog. Nucl. Energy 53 (2011) 139-160.

[32] P. Manneville, Structures dissipatives, chaos et turbulence, Alea Saclay, 1991.

[33] MATLAB®, Copyright 1984-2014, The MathWorks, Inc.

[34] M. Medale, B. Cochelin, High performance computations of steady-state bifurcations in 3D incompressible fluid flows by asymptotic numerical method, J. Comput. Phys. 299 (2015) 581-596.

[35] K. Meerbergen, A. Spence, Implicitly restarted Arnoldi with purification for Shift-Invert transformation, Math. Comput. 66 (1997) $667-689$.

[36] C.B. Moler, G.W. Stewart, An algorithm for generalized matrix eigenvalue problems, SIAM J. Numer. Anal. 10 (1973) 241-256.

[37] H. Muller-Steinhagen, K. Heck, A simple friction pressure drop correlation for two-phase flow in pipes, Chem. Eng. Prog. 20 (1986) $297-308$.

[38] X. Nicolas, M. Medale, S. Glockner, S. Gounand, Benchmark solution for a three-dimensional mixed-convection flow, Part: reference solutions, Numer. Heat Transf., Part B, Fundam. 60 (2011) 325-345.

[39] W.L. Oberkampf, T. Trucano, Verification, and validation in computational fluid dynamics, Prog. Aerosp. Sci. 38 (2002) $209-272$.

[40] V. Pandey, S. Singh, Bifurcation analysis of density wave oscillations in natural circulation loop, Int. J. Therm. Sci. 120 (2017) 446-458.

[41] B. Parisse, R. De Graeve, Giac/Xcas, version 1.5.0, http://www-fourier.ujf-grenoble.fr/ parisse/giac.html, 2018.

[42] Rizwan-Uddin, J.J. Dorning, Some nonlinear dynamics of a heated channel, Nucl. Eng. Des. 93 (1) (1986) 1-14.

[43] P.J. Roache Perspective, A method for uniform reporting of grid refinement studies, J. Fluids Eng. 116 (1994) 405-413.

[44] C.J. Roy, Review of code and solution verification procedures for computational simulation, J. Comput. Phys. 205 (2005) 131-156.

[45] J. Rommes, Arnoldi and Jacobi-Davidson methods for generalized eigenvalue problems $A x=\lambda B x$ with singular B, Math. Comput. 77 (262) (2008) 995-1015.

[46] L.C. Ruspini, C.P. Marcel, A. Clausse, Two-phase flow instabilities: a review, Int. J. Heat Mass Transf. 71 (2014) 521-548.

[47] P. Saha, N. Zuber, An analytical study of the thermally induced two-phase flow instabilities including the effect of thermal non-equilibrium, Int. J. Heat Mass Transf. 21 (4) (1978) 415-426.

[48] R. Seydel, Practical Bifurcation and Stability Analysis, Springer-Verlag, 1994.

[49] R. Seydel, Nonlinear computation, J. Franklin Inst. 334B (1997) 1015-1047.

[50] H.G. Sonnenburg, Full-range drift-flux model based on the combination of drift-flux theory with envelope theory, in: Int. NURETH-4 Conf., Oct. 10-13, 1989, Karlsruhe, 1989, pp. 1003-1009.

[51] L. Sun, K. Mishima, Evaluation analysis of prediction methods for two-phase flow pressure drop in mini-channels, Int. J. Multiph. Flow 35 (2009) $47-54$.

[52] M. Van Dyke, Analysis and improvement of perturbation series, J. Mech. Appl. Math. 27 (1974) 423-450.

[53] D.S. Watkins, Performance of the QZ algorithm in the presence of infinite eigenvalues, SIAM J. Matrix Anal. Appl. 22 (2000) 364-375.

[54] Wolfram Research, Inc. Mathematica, Version 11.3, Champaign, Illinois, 2018.

[55] G.B. Wallis, J.H. Heasley, Oscillations in two-phase flow systems, Trans. ASME J. Heat Transf. 83C (1961) 363-369.

[56] A. Xu, C. Wang, Some extensions of Faà di Bruno's formula with divided differences, Comput. Math. Appl. 59 (6) (2010) $2047-2052$.

[57] Y. Xu, X. Fang, X. Su, Z. Zhou, W. Chen, Evaluation of frictional pressure drop correlations for two-phase flow in pipes, Nucl. Eng. Des. 253 (2012) $86-97$.

[58] P. Yi, S. Yang, C. Habchi, R. Lugo, A multicomponent real-fluid fully compressible four-equation model for two-phase flow with phase-change, Phys. Fluids 31 (2019) 026102.

[59] N. Zuber, J. Findlay, Average volumetric concentration in two-phase flow system, J. Heat Transf. 87 (1965) 453-468. 